\title{
Chiral Proton Catalysis: Enantioselective Brønsted Acid Catalyzed Additions of Nitroacetic Acid Derivatives as Glycine Equivalents
}

\author{
Anand Singh, Ryan A. Yoder, Bo Shen, and Jeffrey N. Johnston* \\ Department of Chemistry, Vanderbilt University \\ 2301 Vanderbilt Place, Nashville, TN 37235-1822
}

\section{Experimental Section}

Flame-dried (under vacuum) glassware was used for all reactions. All reagents and solvents were commercial grade and purified prior to use when necessary. Diethyl ether $\left(\mathrm{Et}_{2} \mathrm{O}\right)$, tetrahydrofuran $(\mathrm{THF})$, dichloromethane $\left(\mathrm{CH}_{2} \mathrm{Cl}_{2}\right)$, and toluene were dried by passage through a column of activated alumina as described by Grubbs. ${ }^{1}$ Methanol was distilled from magnesium under $\mathrm{N}_{2}$ immediately before use. Imines, ${ }^{2} \mathrm{Pd}(\mathrm{dba})_{2},{ }^{3} 9$ anthracenylboronic acid, ${ }^{4}$ and tert-butyl nitroacetate ${ }^{5}$ were prepared as reported in the literature. Buchwald's protocol was used for palladium-mediated aryl amination. ${ }^{6}$

Thin layer chromatography (TLC) was performed using glass-backed silica gel (250 $\mu \mathrm{m})$ plates and flash chromatography utilized 230-400 mesh silica gel from Scientific Adsorbents. UV light, and/or the use of ceric ammonium molybdate and potassium iodoplatinate solutions were used to visualize products.

IR spectra were recorded on a Thermo Nicolet IR100 spectrophotometer and are reported in wavenumbers $\left(\mathrm{cm}^{-1}\right)$. Liquids and oils were analyzed as neat films on a $\mathrm{NaCl}$ plate (transmission), whereas solids were applied to a diamond plate (ATR). Nuclear magnetic resonance spectra (NMR) were acquired on either a Bruker DRX-400 (400 $\mathrm{MHz})$ or DRX-500 (500 MHz) instrument. Chemical shifts are measured relative to residual solvent peaks as an internal standard set to $\delta 7.26$ and $\delta 77.1\left(\mathrm{CDCl}_{3}\right)$. Mass spectra were recorded on a Kratos MS-80 spectrometer by use of chemical ionization (CI). The absolute and relative configuration of syn-6a were determined by X-ray

\footnotetext{
${ }^{1}$ Pangborn, A. B.; Giardello, M. A.; Grubbs, R. H.; Rosen, R. K.; Timmers, F. J. Organometallics 1996, $15,1518-1520$

${ }^{2}$ Kanazawa, A. M.; Denis, J.; Greene, A. E. J. Org. Chem. 1994, 59, 1238-1240.

${ }^{3}$ Rettig, M. F.; Maitlis, P. M. Inorg. Synth. 1992, 28, 110.

${ }^{4}$ Li, Z. H.; Wong, M. S.; Tao, Y.; D’lorio, M. J. Org. Chem. 2004, 69, 921.

${ }^{5}$ Sylvain, C.; Wagner, A.; Mioskowski, C. Tetrahedron Lett. 1999, 40, 875.

${ }^{6}$ Wagaw, S.; Rennels, R.; Buchwald, S. J. Am. Chem. Soc. 1997, 119, 8451-8458.
} 
diffraction. This established the configuration of anti-6a via epimerization. Absolute and relative configuration of the remaining products were assigned by analogy.

General Procedure for the Synthesis of Amines anti-6. A solution of imine 4 (1.0 equiv) and $\mathrm{H}$,Quin $\left({ }^{6}\left({ }^{9} \text { Anth }\right)^{2} \mathrm{Pyr}\right)-\mathrm{BAM} \cdot \mathrm{HOTf}$ (2) (0.05 equiv) in toluene $(0.3 \mathrm{M})$ was cooled to $-78{ }^{\circ} \mathrm{C}$ and treated with tert-butyl nitroacetate (1.1 equiv). The reaction was stirred at $-78^{\circ} \mathrm{C}$ until complete as determined by TLC. The solution was concentrated at $0{ }^{\circ} \mathrm{C}$ and the product was immediately subjected to reduction. The diastereomer ratio of the adduct was determined by ${ }^{1} \mathrm{H}$ NMR.

General Procedure for the Reduction of Aza-Henry Products. A solution of the nitroacetate adduct (1.0 equiv) and cobalt(II) chloride (1.0 equiv) in $\mathrm{MeOH}(0.1 \mathrm{M})$ was cooled to $0{ }^{\circ} \mathrm{C}$ followed by the addition of sodium borohydride (5.0 equiv). The resulting black suspension was stirred at $0{ }^{\circ} \mathrm{C}$ for 15 minutes and then at room temperature until complete (monitored by TLC). The reaction was quenched by the dropwise addition of 3 $\mathrm{M}$ aq $\mathrm{HCl}$ until $\mathrm{pH} 2$ was reached. Then $1 \mathrm{M}$ aq $\mathrm{NH}_{4} \mathrm{OH}$ was added dropwise until the solution attained $\mathrm{pH}$ 9. Methanol was removed, and the aqueous layer was extracted with ethyl acetate. The combined organic extracts were washed with brine, water, and then dried over magnesium sulfate. Filtration and concentration afforded the crude product which was subjected to purification by column chromatography.

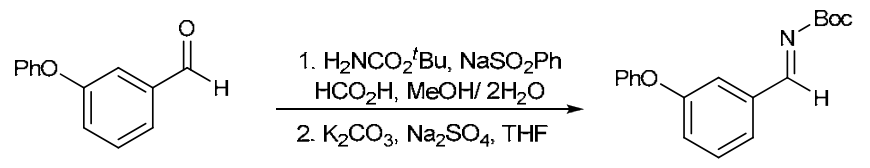

tert-Butyl 3-phenoxybenzylidenecarbamate (4g). Following the Greene protocol, the Schiff base was obtained as a colorless oil. IR (neat) 2977, 1715, 1580, 1487, 1239, 1157 $\mathrm{cm}^{-1}$; ${ }^{1} \mathrm{H}$ NMR $\left(500 \mathrm{MHz}, \mathrm{CDCl}_{3}\right) \delta 8.78(\mathrm{~s}, 1 \mathrm{H}), 7.61(\mathrm{~d}, J=7.7 \mathrm{~Hz}, 1 \mathrm{H}), 7.55(\mathrm{~s}, 1 \mathrm{H})$, $7.43(\mathrm{t}, J=7.9 \mathrm{~Hz}, 1 \mathrm{H}), 7.35$ (dd, $J=7.7,7.7 \mathrm{~Hz}, 2 \mathrm{H}), 7.22-7.20$ (m, 1H), 7.14 (dd, $J=$ 7.4, $7.4 \mathrm{~Hz}, 1 \mathrm{H}), 7.01(\mathrm{~d}, J=8.1 \mathrm{~Hz}, 2 \mathrm{H}), 1.57(\mathrm{~s}, 9 \mathrm{H}) ;{ }^{13} \mathrm{C} \mathrm{NMR}\left(125 \mathrm{MHz}, \mathrm{CDCl}_{3}\right)$ ppm 168.8, 162.5, 158.1, 156.6, 136.0, 130.3, 130.0, 125.4, 124.0, 123.9, 119.3, 119.1, 82.5, 28.0; HRMS (CI): Exact mass calculated for $\mathrm{C}_{18} \mathrm{H}_{20} \mathrm{NO}_{3}[\mathrm{M}+\mathrm{H}]^{+}$298.1438. Found 298.1437 . 


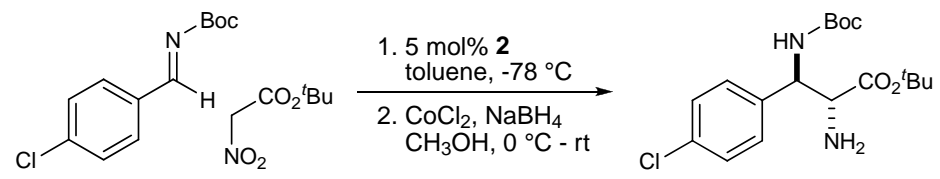

(2R,3R)-tert-Butyl 2-amino-3-(tert-butoxycarbonylamino)-3-(4-

chlorophenyl)propanoate (anti-6a). According to the general procedure, tert-butyl 4chlorobenzylidenecarbamate (4a) $(46.9 \mathrm{mg}, 0.20 \mathrm{mmol})$ provided 6a after flash column chromatography ( $40 \%$ ethyl acetate in hexanes) as a yellow oil $(63.8 \mathrm{mg}, 88 \%$ ), which was determined to be $95 \%$ ee, 5:1 dr by chiral HPLC analysis (Chiralcel AD, 10\% ${ }^{i} \mathrm{PrOH} / \mathrm{hexanes}, 1 \mathrm{~mL} / \mathrm{min}, t_{\mathrm{r}}($ anti, major $)=14.8 \mathrm{~min}, t_{\mathrm{r}}($ anti, minor $)=9.7 \mathrm{~min}, t_{\mathrm{r}}(\mathrm{syn}$, major $)=19.7 \min , t_{\mathrm{r}}($ syn, minor $\left.)=11.8 \mathrm{~min}\right)$.

anti-6a: $\mathrm{R}_{f}=0.07$ (20\% EtOAc/hexanes); IR (neat) 3394, 2975, 1713, 1511, $1155 \mathrm{~cm}^{-1}$; ${ }^{1} \mathrm{H}$ NMR (500 MHz, $\left.\mathrm{CDCl}_{3}\right) \delta$ 7.28-7.21 (m, 4H), 5.89 (br d, $\left.J=7.7 \mathrm{~Hz}, 1 \mathrm{H}\right), 5.02(\mathrm{br} \mathrm{s}$, $1 \mathrm{H}), 3.67$ (br d, $J=4.0 \mathrm{~Hz}, 1 \mathrm{H}), 1.47$ (br s, $2 \mathrm{H}), 1.41$ (s, 9H), $1.38(\mathrm{~s}, 9 \mathrm{H}) ;{ }^{13} \mathrm{C} \mathrm{NMR}$ (125 MHz, $\mathrm{CDCl}_{3}$ ) ppm 172.1, 155.1, 137.2, 133.7, 128.7, 128.5, 82.3, 79.8, 58.6, 55.5, 28.5, 28.1; HRMS (CI): Exact mass calculated for $\mathrm{C}_{18} \mathrm{H}_{28} \mathrm{ClN}_{2} \mathrm{O}_{4}[\mathrm{M}+\mathrm{H}]^{+} 371.1732$. Found 371.1734 .

syn-6a: $\mathrm{R}_{f}=0.10$ (20\% EtOAc/hexanes); IR (neat) 3381, 2981, 1711, 1507, $1156 \mathrm{~cm}^{-1}$; ${ }^{1} \mathrm{H}$ NMR (500 MHz, $\left.\mathrm{CDCl}_{3}\right) \delta$ 7.32-7.26 (m, 4H), $5.72(\mathrm{br} \mathrm{d}, J=1.9 \mathrm{~Hz}, 1 \mathrm{H}), 5.13$ (br d, $J=1.9 \mathrm{~Hz}, 1 \mathrm{H}), 3.74$ (br s, 1H), 1.47 (s, 9H), 1.40 (s, 9H), 1.34 (s, 2H); ${ }^{13} \mathrm{C}$ NMR (125 $\left.\mathrm{MHz}, \mathrm{CDCl}_{3}\right) \mathrm{ppm} 171.3,155.0,139.1,133.1,128.6,127.9,82.3,79.5,58.7,55.8,28.3$, 27.9; HRMS (CI) Exact mass calculated for $\mathrm{C}_{18} \mathrm{H}_{28} \mathrm{ClN}_{2} \mathrm{O}_{4}[\mathrm{M}+\mathrm{H}]^{+}$371.1738. Found 371.1720 .

Anal. Calcd for $\mathrm{C}_{18} \mathrm{H}_{27} \mathrm{ClN}_{2} \mathrm{O}_{4}$ : C, 58.29; H, 7.34; N, 7.55. Found C, 58.04; H, 7.34; N, 7.47 .

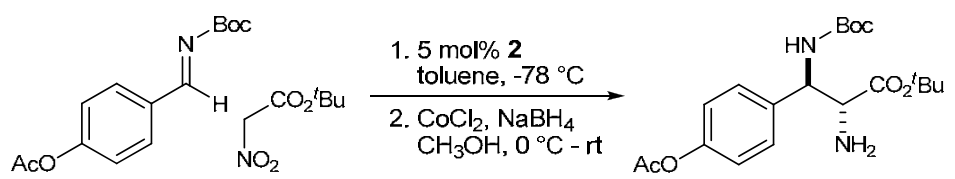

(2R,3R)-tert-Butyl-3-(4-acetoxyphenyl)-2-amino-3-(tert-butoxycarbonylamino)

propanoate (anti-6b). According to the general procedure, tert-butyl 4acetoxybenzylidenecarbamate $(\mathbf{4 b})(50 \mathrm{mg}, 0.18 \mathrm{mmol})$ provided $\mathbf{6 b}$ after flash column 
chromatography (20-40\% ethyl acetate in hexanes) as a colorless oil (55.4 $\mathrm{mg}, 74 \%)$, which was determined to be $89 \%$ ee, 11:1 dr by chiral HPLC analysis (Chiralcel AD, $10 \%{ }^{i} \mathrm{PrOH} /$ hexanes, $1 \mathrm{~mL} / \mathrm{min}, t_{\mathrm{r}}($ anti, major $)=19.7 \mathrm{~min}, t_{\mathrm{r}}($ anti, minor $)=14.5 \mathrm{~min}$, $t_{\mathrm{r}}($ syn, major $)=23.1 \mathrm{~min}, t_{\mathrm{r}}($ syn, minor $\left.)=16.2 \mathrm{~min}\right)$. anti-6b: $\mathrm{R}_{f}=0.13(40 \%$ EtOAc/hexanes); IR (neat) 3404, 2977, 1761, 1713, 1505, 1367, $1198 \mathrm{~cm}^{-1}$; ${ }^{1} \mathrm{H}$ NMR $\left(500 \mathrm{MHz}, \mathrm{CDCl}_{3}\right) \delta 7.29(\mathrm{~d}, J=8.0 \mathrm{~Hz}, 2 \mathrm{H}), 7.02(\mathrm{~d}, J=8.5 \mathrm{~Hz}, 2 \mathrm{H}), 5.88(\mathrm{br} \mathrm{d}, \mathrm{J}=8.1$ $\mathrm{Hz}, 1 \mathrm{H}$ ), 5.05 (br s, 1H), 3.67 (br d, $J=3.5 \mathrm{~Hz}, 1 \mathrm{H}$ ), 2.26 (s, 3H), 1.53 (br s, 2H), 1.41 (s, 9H), 1.35 (s, 9H); ${ }^{13} \mathrm{C} \mathrm{NMR} \mathrm{(125} \mathrm{MHz,} \mathrm{CDCl}_{3}$ ) ppm 172.2, 169.3, 155.1, 150.2, 136.1, 128.2, 121.4, 82.2, 79.7, 58.8, 55.7, 28.4, 28.0, 21.2; HRMS (CI) Exact mass calculated for $\mathrm{C}_{20} \mathrm{H}_{31} \mathrm{~N}_{2} \mathrm{O}_{6}[\mathrm{M}+\mathrm{H}]^{+}$395.2177. Found 395.2182.

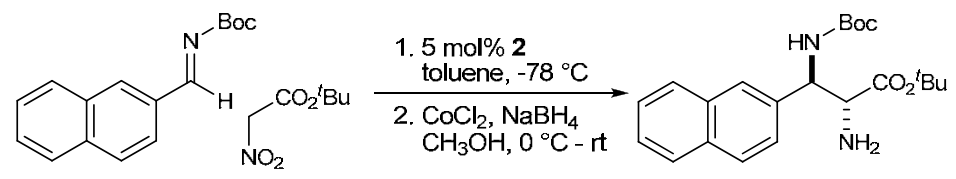

(2R,3R)-tert-Butyl-2-amino-3-(tert-butoxycarbonylamino)-3-(naphthalen-2-yl)

propanoate (anti-6c). According to the general procedure, tert-butyl naphthalen-2ylmethylenecarbamate (4c) $(50 \mathrm{mg}, 0.20 \mathrm{mmol}$ ) provided 6c after flash column chromatography (20-40\% ethyl acetate in hexanes) as a colorless oil $(60.5 \mathrm{mg}, 80 \%)$, which was determined to be $91 \%$ ee, 11:1 dr by chiral HPLC analysis (Chiralcel AD, $10 \%{ }^{i} \mathrm{PrOH} /$ hexanes, $1 \mathrm{~mL} / \mathrm{min}, t_{\mathrm{r}}($ anti, major $)=17.8 \mathrm{~min}, t_{\mathrm{r}}($ anti, minor $)=12.5 \mathrm{~min}$, $t_{\mathrm{r}}($ syn, major $)=26.9 \mathrm{~min}, t_{\mathrm{r}}($ syn, minor $\left.)=14.5 \mathrm{~min}\right)$. anti-6c: $\mathrm{R}_{f}=0.23(40 \%$ EtOAc/hexanes); IR (neat) 3393, 2976, 2926, 1712, 1493, 1367, $1249 \mathrm{~cm}^{-1}$; ${ }^{1} \mathrm{H}$ NMR (500 MHz, $\left.\mathrm{CDCl}_{3}\right) \delta$ 7.79-7.74 (m, 4H), 7.46-7.42 (m, 3H), $6.03($ br d, $J=7.9 \mathrm{~Hz}, 1 \mathrm{H})$, 5.23 (br s, 1H), 3.77 (br d, $J=3.8 \mathrm{~Hz}, 1 \mathrm{H}), 1.49$ (s, 2H), 1.43 (s, 9H), 1.37 (s, 9H); ${ }^{13} \mathrm{C}$ NMR (125 MHz, $\left.\mathrm{CDCl}_{3}\right)$ ppm 172.3, 155.2, 136.0, 133.2, 133.1, 128.2, 128.0, 127.7, 126.4, 126.2, 125.9, 125.2, 82.1, 79.7, 59.0, 56.3, 28.4, 28.1; HRMS (CI): Exact mass calculated for $\mathrm{C}_{22} \mathrm{H}_{31} \mathrm{~N}_{2} \mathrm{O}_{4}[\mathrm{M}+\mathrm{H}]^{+}$387.2284. Found 387.2278.

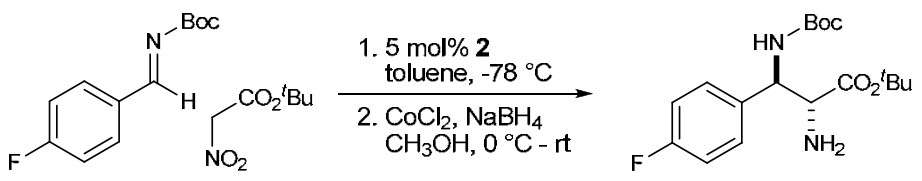

(2R,3R)-tert-Butyl-2-amino-3-(tert-butoxycarbonylamino)-3-(4-

florophenyl)propanoate (anti-6d). According to the general procedure, tert-butyl 4florobenzylidenecarbamate (4d) $(44.6 \mathrm{mg}, 0.20 \mathrm{mmol})$ provided $6 \mathbf{d}$ after flash column 
chromatography (40\% ethyl acetate in hexanes) as a yellow oil (57.3 $\mathrm{mg}, 81 \%$ ), which was determined to be $93 \%$ ee, 6:1 dr by chiral HPLC analysis (Chiralcel AD, 10\% ${ }^{i} \mathrm{PrOH} / \mathrm{hexanes}, 1 \mathrm{~mL} / \mathrm{min}, t_{\mathrm{r}}($ anti, major $)=11.8 \mathrm{~min}, t_{\mathrm{r}}($ anti, minor $)=8.1 \mathrm{~min}, t_{\mathrm{r}}($ syn, major $)=15.1 \mathrm{~min}, t_{\mathrm{r}}($ syn, minor $\left.)=10.2 \mathrm{~min}\right)$. anti-6d: $\mathrm{R}_{f}=0.10(20 \%$ EtOAc/hexanes $)$; IR (neat) 3411, 2976, 2924, 1712, 1508, $1157 \mathrm{~cm}^{-1}$; ${ }^{1} \mathrm{H}$ NMR (500 MHz, $\mathrm{CDCl}_{3}$ ) $\delta 7.30-$ 7.24 (m, 2H), 6.98 (t, $J=8.7 \mathrm{~Hz}, 2 \mathrm{H}$ ), 5.89 (br s, 1H), 5.03 (br s, 1H), 3.66 (br s, 1H), $1.46(\mathrm{~s}, 2 \mathrm{H}), 1.41$ (s, 9H), 1.37 (s, 9H); ${ }^{13} \mathrm{C}$ NMR $\left(125 \mathrm{MHz}, \mathrm{CDCl}_{3}\right) \mathrm{ppm} 172.2,162.4$ $\left(\mathrm{d},{ }^{1} J_{\mathrm{CF}}=244.4 \mathrm{~Hz}\right), 155.1,134.4,128.9\left(\mathrm{~d},{ }^{3} J_{\mathrm{CF}}=7.9 \mathrm{~Hz}\right), 115.2\left(\mathrm{~d},{ }^{2} J_{\mathrm{CF}}=21.7 \mathrm{~Hz}\right)$, 82.2, 79.7, 58.7, 55.4, 28.5, 28.1; ${ }^{19} \mathrm{~F}$ NMR (282 $\left.\mathrm{MHz}, \mathrm{CDCl}_{3}\right)$ ppm -113.2; HRMS (CI): Exact mass calculated for $\mathrm{C}_{18} \mathrm{H}_{28} \mathrm{FN}_{2} \mathrm{O}_{4}[\mathrm{M}+\mathrm{H}]^{+}$355.2028. Found 355.2035.

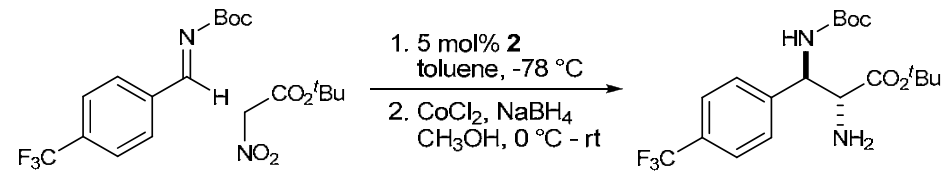

(2R,3R)-tert-Butyl-2-amino-3-(tert-butoxycarbonylamino)-3-(4-

(trifluoromethyl)phenyl) propanoate (anti-6e). According to the general procedure, tert-butyl 4-trifluoromethylbenzylidenecarbamate (4e) $(50 \mathrm{mg}, 0.18 \mathrm{mmol})$ provided $\mathbf{6 e}$ after flash column chromatography (20-40\% ethyl acetate in hexanes) as a yellow oil (61.4 mg, 83\%), which was determined to be $88 \%$ ee, $7: 1 \mathrm{dr}$ by chiral HPLC analysis $\left(\right.$ Chiralcel AD, $3 \%{ }^{i} \mathrm{PrOH} /$ hexanes, $1 \mathrm{~mL} / \mathrm{min}, t_{\mathrm{r}}($ anti, major $)=57.8 \mathrm{~min}, t_{\mathrm{r}}($ anti, minor $)=$ $32.5 \mathrm{~min}, t_{\mathrm{r}}($ syn, major $)=69.5 \mathrm{~min}, t_{\mathrm{r}}($ syn, minor $\left.)=41.7 \mathrm{~min}\right)$. anti-6e: $\mathrm{R}_{f}=0.26(40 \%$ EtOAc/hexanes); IR (neat) 3383, 2977, 2925, 1714, 1492, 1368, 1326, 1297, $1161 \mathrm{~cm}^{-1}$; ${ }^{1} \mathrm{H}$ NMR $\left(500 \mathrm{MHz}, \mathrm{CDCl}_{3}\right) \delta 7.56(\mathrm{~d}, J=8.0 \mathrm{~Hz}, 2 \mathrm{H}), 7.42(\mathrm{~d}, J=7.7 \mathrm{~Hz}, 2 \mathrm{H}), 5.95(\mathrm{br}$ d, $J=7.6 \mathrm{~Hz}, 1 \mathrm{H}$ ), 5.10 (br s, 1H), 3.70 (br d, $J=2.7 \mathrm{~Hz}, 1 \mathrm{H}), 1.48$ (s, 2H), 1.42 (s, 9H), $1.38(\mathrm{~s}, 9 \mathrm{H}) ;{ }^{13} \mathrm{C}$ NMR $\left(125 \mathrm{MHz}, \mathrm{CDCl}_{3}\right) \mathrm{ppm} 172.0,155.1,142.8,130.0\left(\mathrm{q},{ }^{2} J_{\mathrm{CF}}=32.5\right.$ $\mathrm{Hz}), 127.7,125.3,124.3\left(\mathrm{q},{ }^{1} J_{\mathrm{CF}}=272.3 \mathrm{~Hz}\right.$ ), 82.5, 80.0, 58.6, 55.8, 28.5, 28.1; HRMS (CI): Exact mass calculated for $\mathrm{C}_{19} \mathrm{H}_{28} \mathrm{~N}_{2} \mathrm{O}_{4} \mathrm{~F}_{3}[\mathrm{M}+\mathrm{H}]^{+}$405.2001. Found 405.1996.

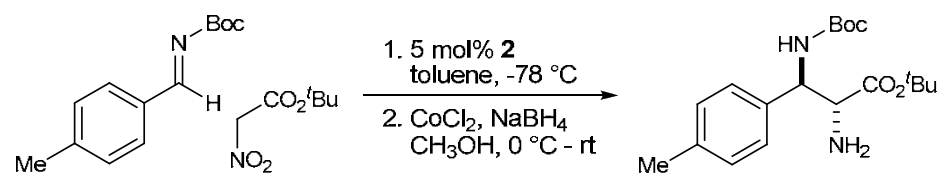

(2R,3R)-tert-Butyl 2-amino-3-(tert-butoxycarbonylamino)-3-p-tolylpropanoate (anti6f). According to the general procedure, tert-butyl 4-fluorobenzylidene carbamate (4f) (40.9 $\mathrm{mg}, 0.19 \mathrm{mmol}$ ) provided $\mathbf{6 f}$ after flash column chromatography ( $40 \%$ ethyl acetate 
in hexanes) as a yellow oil (52.9 mg, 81\%), which was determined to be $95 \%$ ee, $6: 1 \mathrm{dr}$ by chiral HPLC analysis (Chiralcel AD, $10 \%{ }^{i} \mathrm{PrOH} /$ hexanes, $1 \mathrm{~mL} / \mathrm{min}, t_{\mathrm{r}}$ (anti, major) $=$ $10.7 \mathrm{~min}, t_{\mathrm{r}}($ anti, minor $)=8.2 \mathrm{~min}, t_{\mathrm{r}}($ syn, major $)=20.4 \mathrm{~min}, t_{\mathrm{r}}($ syn, minor $\left.)=12.8 \mathrm{~min}\right)$. anti-6f: $\mathrm{R}_{f}=0.25$ (40\% EtOAc/hexanes); IR (neat) 3410, 2977, 2922, 1713, 1492, 1157, $732 \mathrm{~cm}^{-1} ;{ }^{1} \mathrm{H}$ NMR (500 MHz, $\left.\mathrm{CDCl}_{3}\right) \delta$ 7.20-7.13 (m, 2H), 7.10-7.07 (m, 2H), 5.85 (br d, $J=7.8 \mathrm{~Hz}, 1 \mathrm{H}$ ), 5.02 (br s, 1H), 3.66 (br s, 1H), 2.30 (s, 3H), 1.47 (s, 2H), 1.41 (s, 9H), 1.39 (s, 9H); ${ }^{13} \mathrm{C}$ NMR (125 MHz, $\mathrm{CDCl}_{3}$ ) ppm 172.5, 155.1, 137.4, 135.4, 129.1, 127.1, 82.0, 79.5, 59.0, 55.9, 28.5, 28.1, 21.2; HRMS (CI): Exact mass calculated for $\mathrm{C}_{19} \mathrm{H}_{31} \mathrm{~N}_{2} \mathrm{O}_{4}[\mathrm{M}+\mathrm{H}]^{+}$351.2278. Found 351.2277.

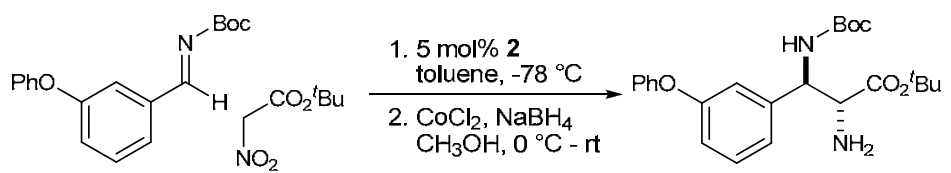

(2R,3R)-tert-Butyl-2-amino-3-(tert-butoxycarbonylamino)-3-(3-phenoxyphenyl)

propanoate (anti-6g). According to the general procedure, tert-butyl 3phenoxybenzylidenecarbamate $(\mathbf{4 g})(50 \mathrm{mg}, 0.17 \mathrm{mmol})$ provided $\mathbf{6 g}$ after flash column chromatography (20-40\% ethyl acetate in hexanes) as a colorless oil $(60.5 \mathrm{mg}, 84 \%)$, which was determined to be $87 \%$ ee, 6:1 dr by chiral HPLC analysis (Chiralcel IA, 5\% $\mathrm{EtOH} /$ hexanes, $1 \mathrm{~mL} / \mathrm{min}, t_{\mathrm{r}}$ (anti, major $)=17.5 \mathrm{~min}, t_{\mathrm{r}}($ anti, minor $)=12.5 \mathrm{~min}, t_{\mathrm{r}}($ syn, major $)=11.5 \mathrm{~min}, t_{\mathrm{r}}($ syn, minor $\left.)=10.0 \mathrm{~min}\right)$. anti-6g: $\mathrm{R}_{f}=0.28(40 \%$ EtOAc/hexanes $)$; IR (neat) 3388, 2977, 2925, 1715, 1584, 1487, 1244, $1158 \mathrm{~cm}^{-1}$; ${ }^{1} \mathrm{H}$ NMR $(500 \mathrm{MHz}$, $\left.\mathrm{CDCl}_{3}\right) \delta 7.35(\mathrm{dd}, J=7.5,7.5 \mathrm{~Hz}, 2 \mathrm{H}), 7.29-7.27(\mathrm{~m}, 1 \mathrm{H}), 7.12(\mathrm{t}, J=7.4 \mathrm{~Hz}, 1 \mathrm{H}), 7.05$ (d, $J=7.7 \mathrm{~Hz}, 1 \mathrm{H}), 7.01-6.99(\mathrm{~m}, 3 \mathrm{H}), 6.90(\mathrm{~d}, J=7.9 \mathrm{~Hz}, 1 \mathrm{H}), 5.90(\mathrm{br} \mathrm{d}, J=7.7 \mathrm{~Hz}$, 1H), 5.07 (br s, 1H), 3.69 (br s, 1H), 1.57 (br s, 2H), 1.45 (s, 9H), 1.40 (s, 9H); ${ }^{13} \mathrm{C}$ NMR (125 MHz, $\mathrm{CDCl}_{3}$ ) ppm 172.3, 157.2, 157.1, 155.1, 140.6, 129.8, 129.6, 123.3, 122.2, 119.0, 118.1, 117.8, 82.2, 79.7, 58.8, 55.9, 28.5, 28.1; HRMS (CI): Exact mass calculated for $\mathrm{C}_{24} \mathrm{H}_{33} \mathrm{~N}_{2} \mathrm{O}_{5}[\mathrm{M}+\mathrm{H}]^{+}$429.2389. Found 429.2384.

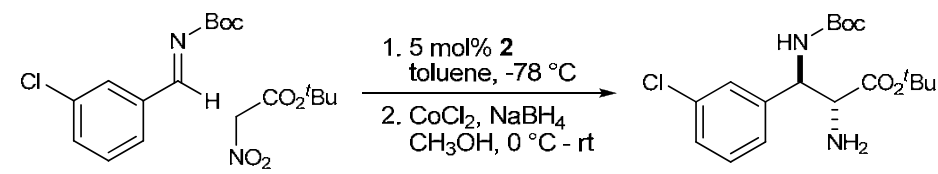

(2R,3R)-tert-Butyl-2-amino-3-(tert-butoxycarbonylamino)-3-(3-chlorophenyl)

propanoate (anti-6h). According to the general procedure, tert-butyl 3chlorobenzylidenecarbamate $(4 \mathbf{h})(50 \mathrm{mg}, 0.21 \mathrm{mmol})$ provided $\mathbf{6 h}$ after flash column 
chromatography (20-40\% ethyl acetate in hexanes) as a colorless oil $(54.1 \mathrm{mg}, 70 \%)$, which was determined to be $87 \%$ ee, 10:1 dr by chiral HPLC analysis (Chiralcel IA, 15\% ${ }^{i} \mathrm{PrOH} / \mathrm{h}$ exanes, $0.5 \mathrm{~mL} / \mathrm{min}, \mathrm{t}_{\mathrm{r}}($ anti, major $)=15.1 \mathrm{~min}, t_{\mathrm{r}}($ anti, minor $)=12.2 \mathrm{~min}, t_{\mathrm{r}}($ syn, major $)=17.4 \mathrm{~min}, t_{\mathrm{r}}($ syn, minor $\left.)=14.1 \mathrm{~min}\right)$. anti-6h: $\mathrm{R}_{f}=0.3(40 \%$ EtOAc/hexanes $)$; IR (neat) 3381, 2977, 2926, 1714, 1481, 1367, 1248, $1155 \mathrm{~cm}^{-1} ;{ }^{1} \mathrm{H}$ NMR (500 MHz, $\left.\mathrm{CDCl}_{3}\right) \delta 7.28(\mathrm{~s}, 1 \mathrm{H}), 7.23-7.22(\mathrm{~m}, 2 \mathrm{H}), 7.18-7.17(\mathrm{~m}, 1 \mathrm{H}), 5.94(\mathrm{br} \mathrm{d}, J=8.0 \mathrm{~Hz}, 1 \mathrm{H})$, 5.03 (br d, $J=3.5 \mathrm{~Hz}, 1 \mathrm{H}$ ), 3.67 (br d, $J=4.1 \mathrm{~Hz}, 1 \mathrm{H}$ ), 1.47 (s, 2H), 1.42 (s, 9H), 1.39 (s, $9 \mathrm{H}) ;{ }^{13} \mathrm{C}$ NMR (125 MHz, $\mathrm{CDCl}_{3}$ ) ppm 171.9, 154.9, 140.5, 134.1, 129.5, 127.8, 127.4, 125.2, 82.3, 79.7, 58.5, 55.5, 28.3, 27.9; HRMS (CI): Exact mass calculated for $\mathrm{C}_{18} \mathrm{H}_{28} \mathrm{ClN}_{2} \mathrm{O}_{4}[\mathrm{M}+\mathrm{H}]^{+}$371.1738. Found 371.1732.

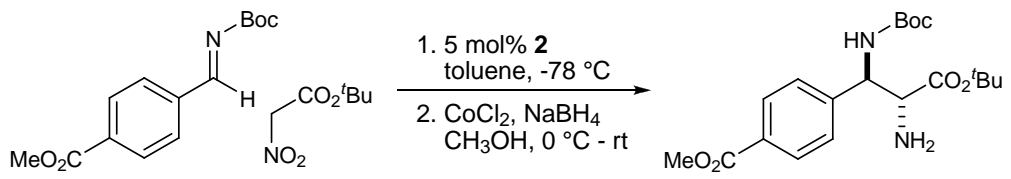

Methyl

4-((1R,2R)-2-Amino-3-tert-butoxy-1-(tert-butoxycarbonylamino)-3-

oxopropyl)benzoate (anti-6i). According to the general procedure, methyl 4-((tertbutoxycarbonylimino)methyl)benzoate $(4 \mathbf{i})(50 \mathrm{mg}, 0.19 \mathrm{mmol})$ provided $\mathbf{6 i}$ after flash column chromatography (20-40\% ethyl acetate in hexanes) as a pale yellow oil $(62.9 \mathrm{mg}$, $84 \%$ ), which was determined to be $95 \%$ ee, $8: 1 \mathrm{dr}$ by chiral HPLC analysis (Chiralcel $\mathrm{AD}, 10 \%{ }^{i} \mathrm{PrOH} /$ hexanes, $1 \mathrm{~mL} / \mathrm{min}, t_{\mathrm{r}}$ (anti, major $)=25.6 \mathrm{~min}, t_{\mathrm{r}}($ anti, minor $)=17.7$ $\min , t_{\mathrm{r}}($ syn, major $)=48.9 \min , t_{\mathrm{r}}($ syn, minor $\left.)=30.7 \mathrm{~min}\right)$. anti-6i: $\mathrm{R}_{f}=0.23(40 \%$ EtOAc/hexanes); IR (neat) 3396, 2978, 1716, 1492, 1281, $1155 \mathrm{~cm}^{-1} ;{ }^{1} \mathrm{H}$ NMR (500 $\left.\mathrm{MHz}, \mathrm{CDCl}_{3}\right) \delta 7.95(\mathrm{~d}, J=8.3 \mathrm{~Hz}, 2 \mathrm{H}), 7.35$ (d, $\left.J=7.9 \mathrm{~Hz}, 2 \mathrm{H}\right), 6.0($ br d, $J=8.3 \mathrm{~Hz}$, 1H), 5.08 (br d, $J=3.4 \mathrm{~Hz}, 1 \mathrm{H}$ ), 3.87 (s, 3H), 3.69 (br d, $J=2.77 \mathrm{~Hz}, 1 \mathrm{H}$ ), 1.54 (br s, 2H), 1.39 (s, 9H), 1.35 (s, 9H); ${ }^{13} \mathrm{C}$ NMR (125 MHz, $\mathrm{CDCl}_{3}$ ) ppm 172.0, 166.8, 155.0, 143.8, 129.6, 127.3, 126.6, 82.3, 79.8, 58.5, 55.9, 52.1, 28.3, 27.9; HRMS (CI): Exact mass calculated for $\mathrm{C}_{20} \mathrm{H}_{31} \mathrm{~N}_{2} \mathrm{O}_{6}[\mathrm{M}+\mathrm{H}]^{+}$395.2182. Found 395.2177.
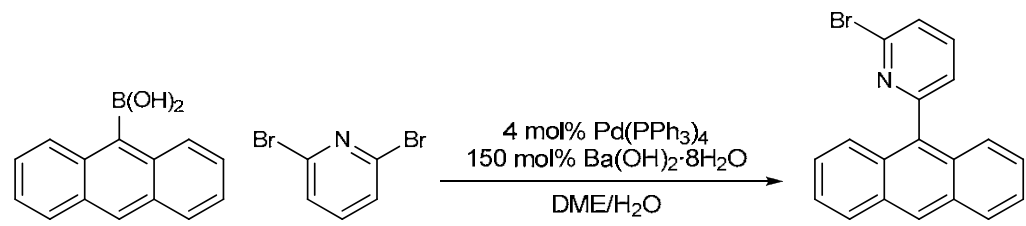

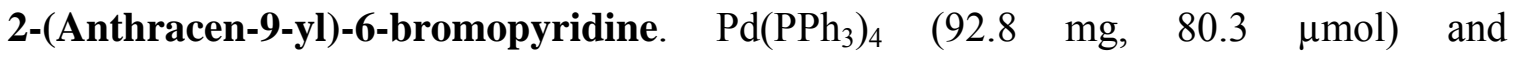
$\mathrm{Ba}(\mathrm{OH})_{2} \cdot 8 \mathrm{H}_{2} \mathrm{O} \quad(950 \mathrm{mg}, 3.01 \mathrm{mmol}) \quad$ were added into the solution of 9- 
anthracenylboronic acid (446 mg, $2.01 \mathrm{mmol})$ in $15 \mathrm{~mL} \mathrm{DME} / \mathrm{H}_{2} \mathrm{O}(2: 1)$, followed by the addition of 2,6-dibromopyridine $(571 \mathrm{mg}, 2.41 \mathrm{mmol})$. The reaction was allowed to stir under reflux for $10 \mathrm{~h}$. After cooling to room temperature, the reaction mixture was extracted with chloroform and the organic layer was dried, filtered, concentrated and purified by flash column chromatography on silica gel (3\% ethyl acetate in hexanes) to give the bromopyridine as a yellow solid (503 $\mathrm{mg}, 75 \%)$. Mp 88-91 ${ }^{\circ} \mathrm{C} ; \mathrm{R}_{f}=0.30(10 \%$ EtOAc/ hexanes). IR (neat) 3056, 2921, 1577, 1546, 1119, 908, $731 \mathrm{~cm}^{-1} ;{ }^{1} \mathrm{H}$ NMR (500 $\left.\mathrm{MHz}, \mathrm{CDCl}_{3}\right) \delta 8.55(\mathrm{~s}, 1 \mathrm{H}), 8.04(\mathrm{~d}, J=9.5 \mathrm{~Hz}, 2 \mathrm{H}), 7.78(\mathrm{t}, J=7.7 \mathrm{~Hz}, 1 \mathrm{H}), 7.67(\mathrm{~d}, J$ $=7.7 \mathrm{~Hz}, 1 \mathrm{H}), 7.57(\mathrm{~d}, J=6.7 \mathrm{~Hz}, 2 \mathrm{H}), 7.49(\mathrm{~d}, J=8.1 \mathrm{~Hz}, 1 \mathrm{H}), 7.46(\mathrm{t}, J=7.7 \mathrm{~Hz}, 2 \mathrm{H})$, 7.42-7.38 (m, 2H); ${ }^{13} \mathrm{C}$ NMR $\left(125 \mathrm{MHz}, \mathrm{CDCl}_{3}\right) \delta 159.5,142.3,138.6,133.3,131.4$, 130.0, 128.6, 128.2, 127.0, 126.3, 125.9, 125.7, 125.3; HRMS (CI): Exact mass calculated for $\mathrm{C}_{19} \mathrm{H}_{13} \mathrm{NBr}[\mathrm{M}+\mathrm{H}]^{+}$334.0226. Found 334.0210.

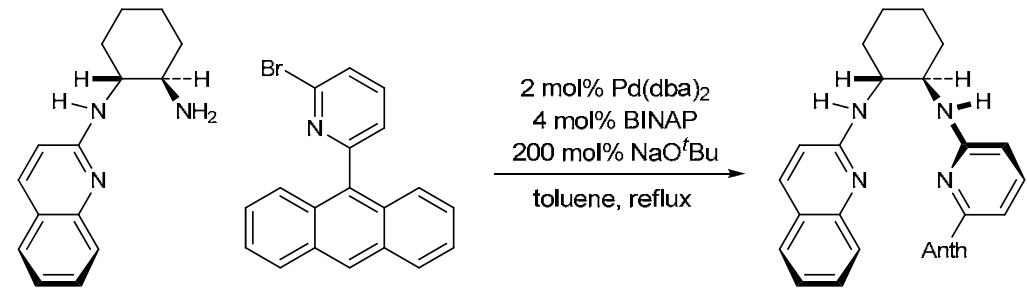

H,Quin $\left({ }^{6}\left({ }^{9} \text { Anth }\right)^{2}\right.$ Pyr $)$-BAM. Pd(dba $)_{2}(6.7 \mathrm{mg}, 12 \mu \mathrm{mol})$, rac-BINAP (14.5 mg, 23.3 $\mu \mathrm{mol})$, and $\mathrm{NaO}^{t} \mathrm{Bu}(112 \mathrm{mg}, 1.17 \mathrm{mmol})$ were loaded into a round bottom flask in a glove box. Toluene $(5.8 \mathrm{~mL})$ was added to the mixture followed by the amine (141 $\mathrm{mg}$, $584 \mu \mathrm{mol})$ and 2-(anthracen-9-yl)-6-bromopyridine (195 mg, $584 \mu \mathrm{mol})$. The reaction was refluxed for $12 \mathrm{~h}$, and then cooled to room temperature, filtered through Celite, concentrated, and purified by flash column chromatography on silica gel (20\% ethyl acetate in hexanes), to afford the desired bis(amidine) as a yellow foam ( $80.0 \mathrm{mg}, 28 \%)$. $[\alpha]_{\mathrm{D}}^{20}+405.1$ (c 1.0, $\mathrm{CHCl}_{3}$ ); $\mathrm{R}_{f}=0.10$ (20\% ethyl acetate/hexanes). IR (neat) 2928, $1618,1599,1518,1452,755 \mathrm{~cm}^{-1} ;{ }^{1} \mathrm{H}$ NMR $\left(500 \mathrm{MHz}, \mathrm{CDCl}_{3}\right) \delta 8.52(\mathrm{~s}, 1 \mathrm{H}), 8.06(\mathrm{t}, J$ $=8.4 \mathrm{~Hz}, 2 \mathrm{H}), 7.82(\mathrm{t}, J=9.6 \mathrm{~Hz}, 2 \mathrm{H}), 7.67(\mathrm{~d}, J=8.6 \mathrm{~Hz}, 1 \mathrm{H}), 7.53-7.35(\mathrm{~m}, 8 \mathrm{H}), 7.16$ (t, $J=7.1 \mathrm{~Hz}, 1 \mathrm{H}), 6.70(\mathrm{~d}, J=7.0 \mathrm{~Hz}, 1 \mathrm{H}), 6.40($ br d, $J=7.3 \mathrm{~Hz}, 1 \mathrm{H}), 6.15$ (br d, $J=$ $8.2 \mathrm{~Hz}, 1 \mathrm{H}$ ), 5.56 (br d, $J=6.0 \mathrm{~Hz}, 1 \mathrm{H}$ ), 5.37 (br d, $J=6.6 \mathrm{~Hz}, 1 \mathrm{H}$ ), 4.04 (br d, $J=8.0$ $\mathrm{Hz}, 1 \mathrm{H}), 3.86$ (br d, $J=8.0 \mathrm{~Hz}, 1 \mathrm{H}), 2.25$ (br t, $J=8.0 \mathrm{~Hz}, 2 \mathrm{H}), 1.71-1.61(\mathrm{~m}, 2 \mathrm{H}), 1.45-$ $1.32(\mathrm{~m}, 4 \mathrm{H}) ;{ }^{13} \mathrm{C}$ NMR $\left(125 \mathrm{MHz}, \mathrm{CDCl}_{3}\right) \delta 158.8,156.8,155.8,148.0,137.0,136.6$, $136.5,131.6,131.5,130.1,129.9,129.3,128.5,128.3,127.4,127.0,126.9,126.8,126.0$, 
125.5, 125.4, 125.2, 125.1, 123.3, 121.6, 115.5, 113.1, 107.4, 55.6, 55.1, 32.7, 32.0, 24.6, 24.4; HRMS (CI): Exact mass calculated for $\mathrm{C}_{34} \mathrm{H}_{31} \mathrm{~N}_{4}[\mathrm{M}+\mathrm{H}]^{+}$495.2543. Found 495.2529 . 


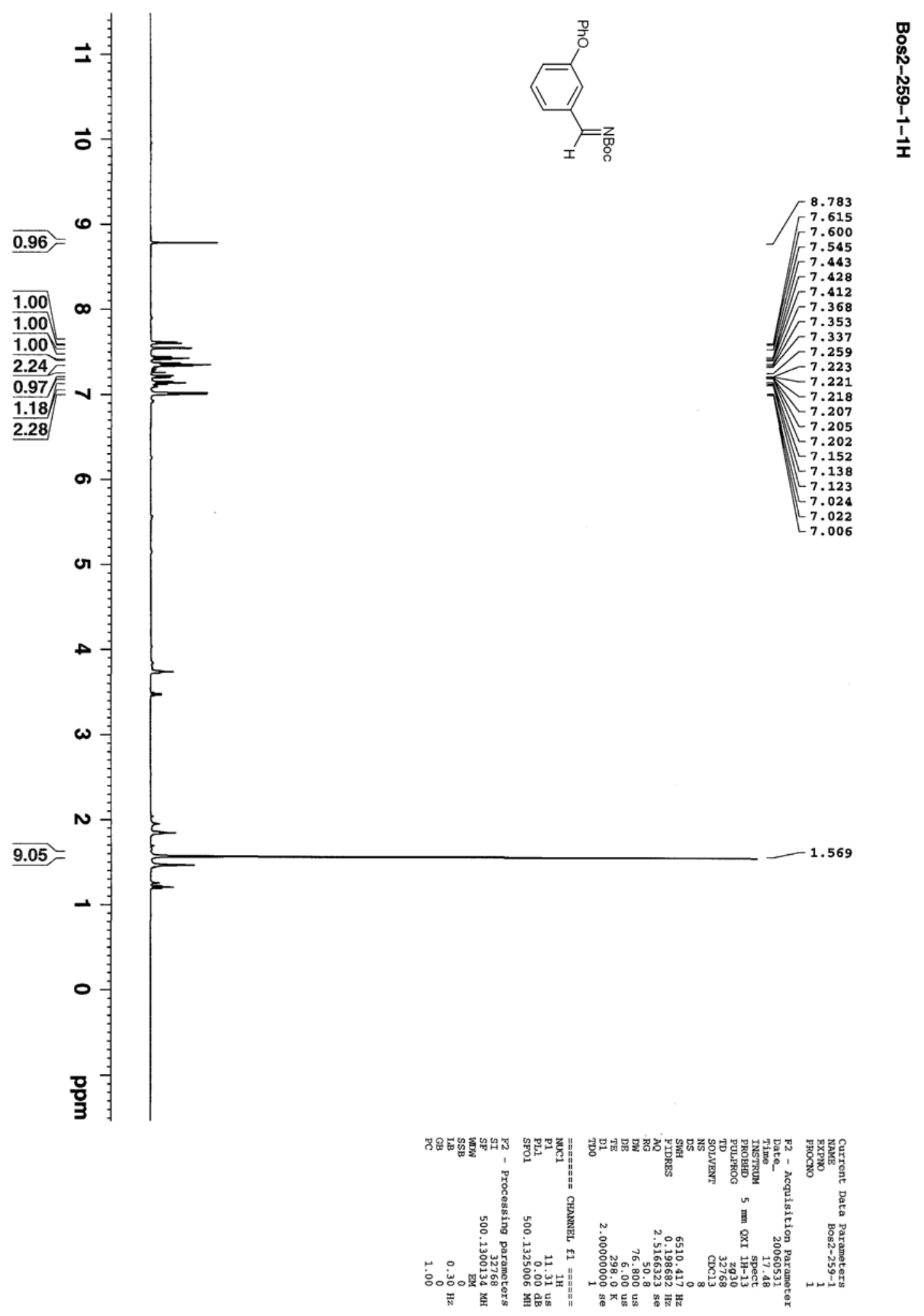




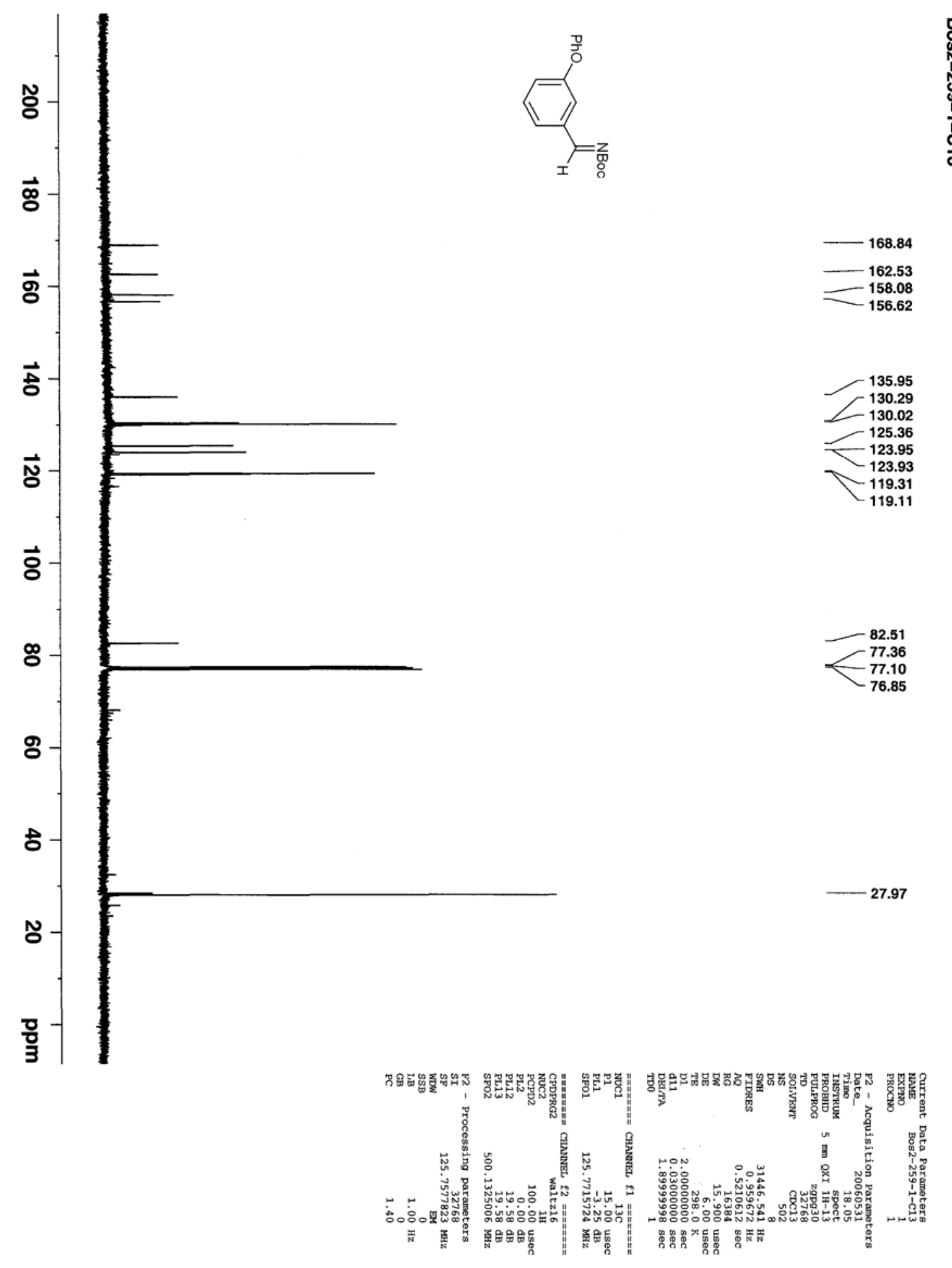




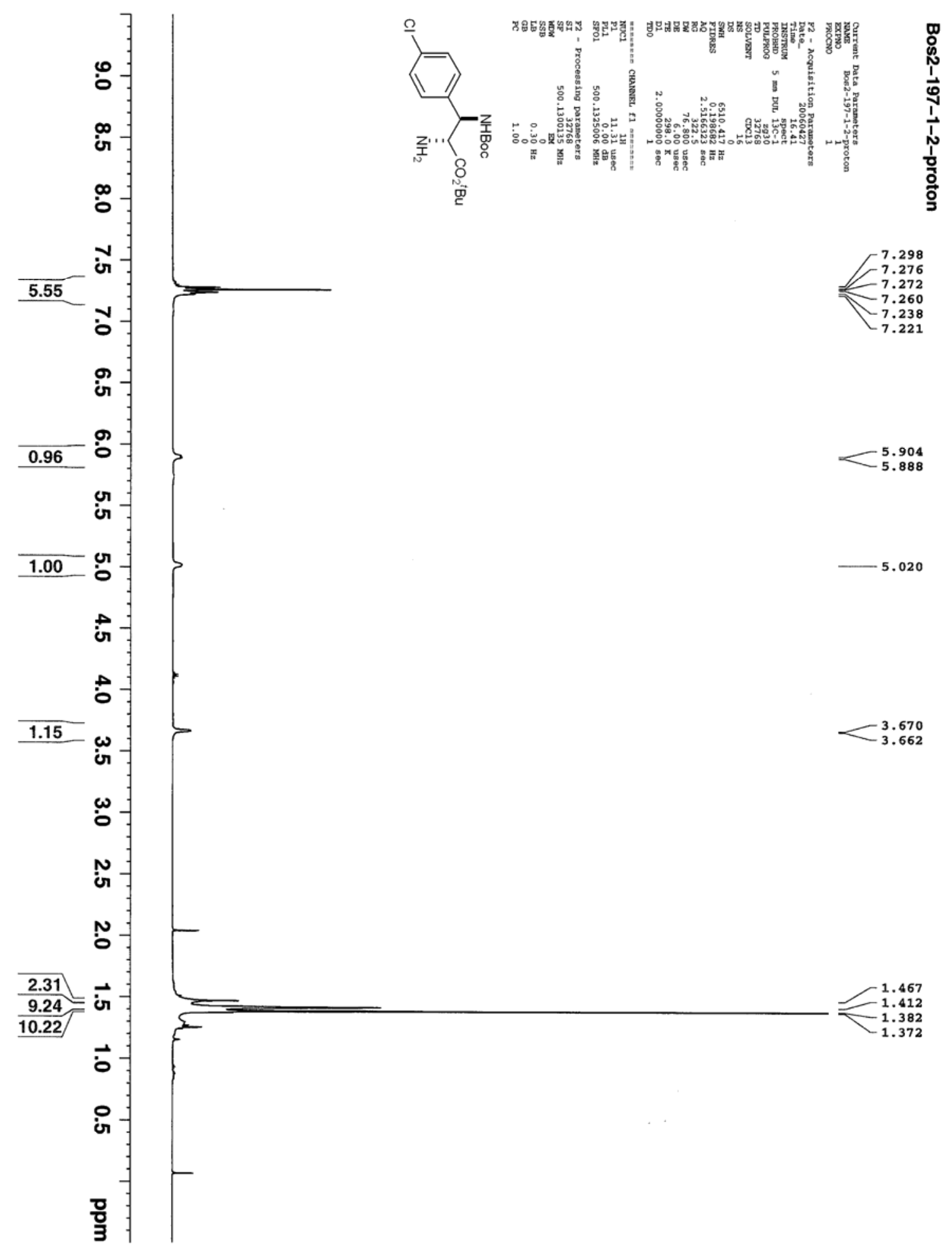




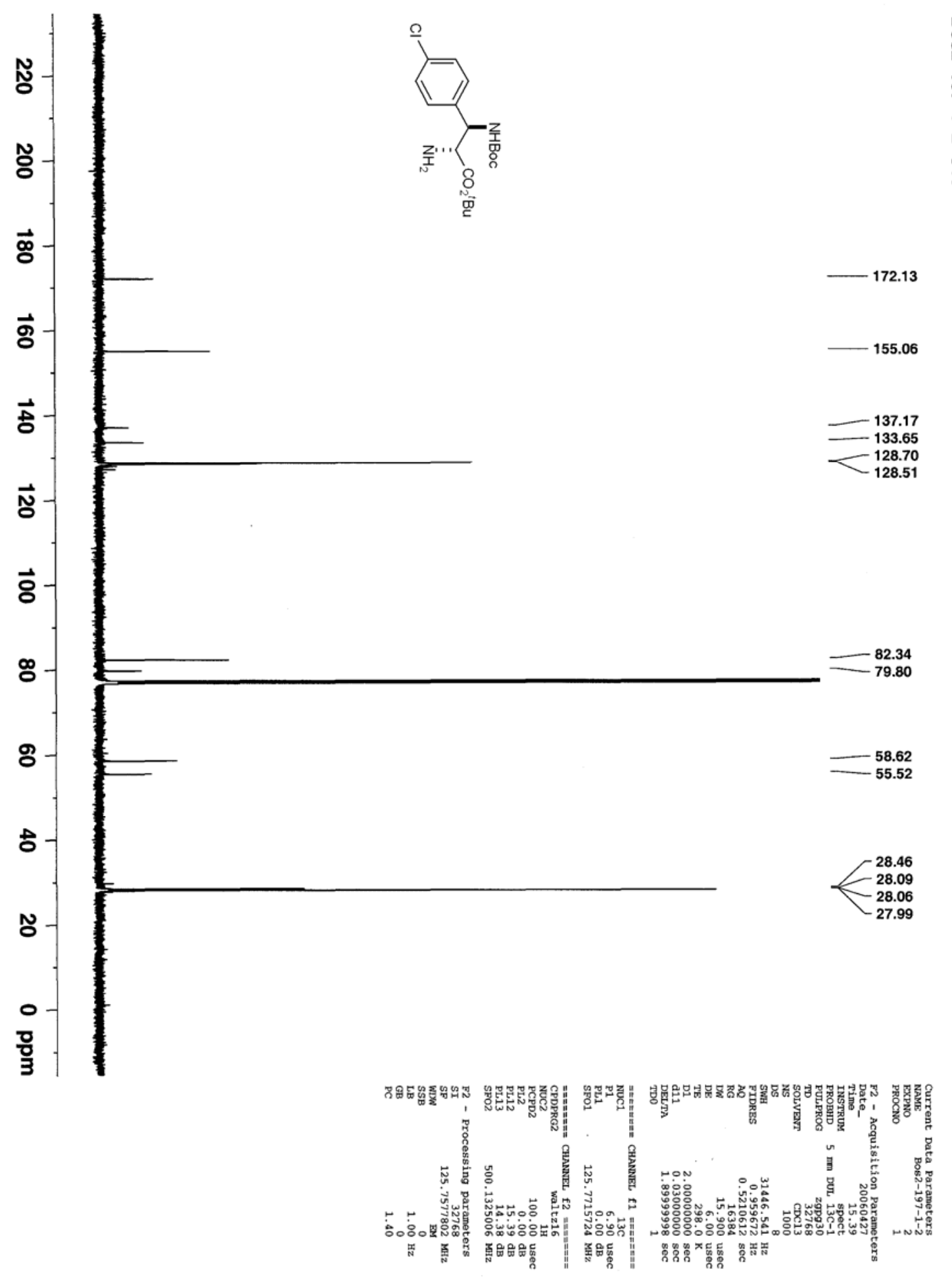




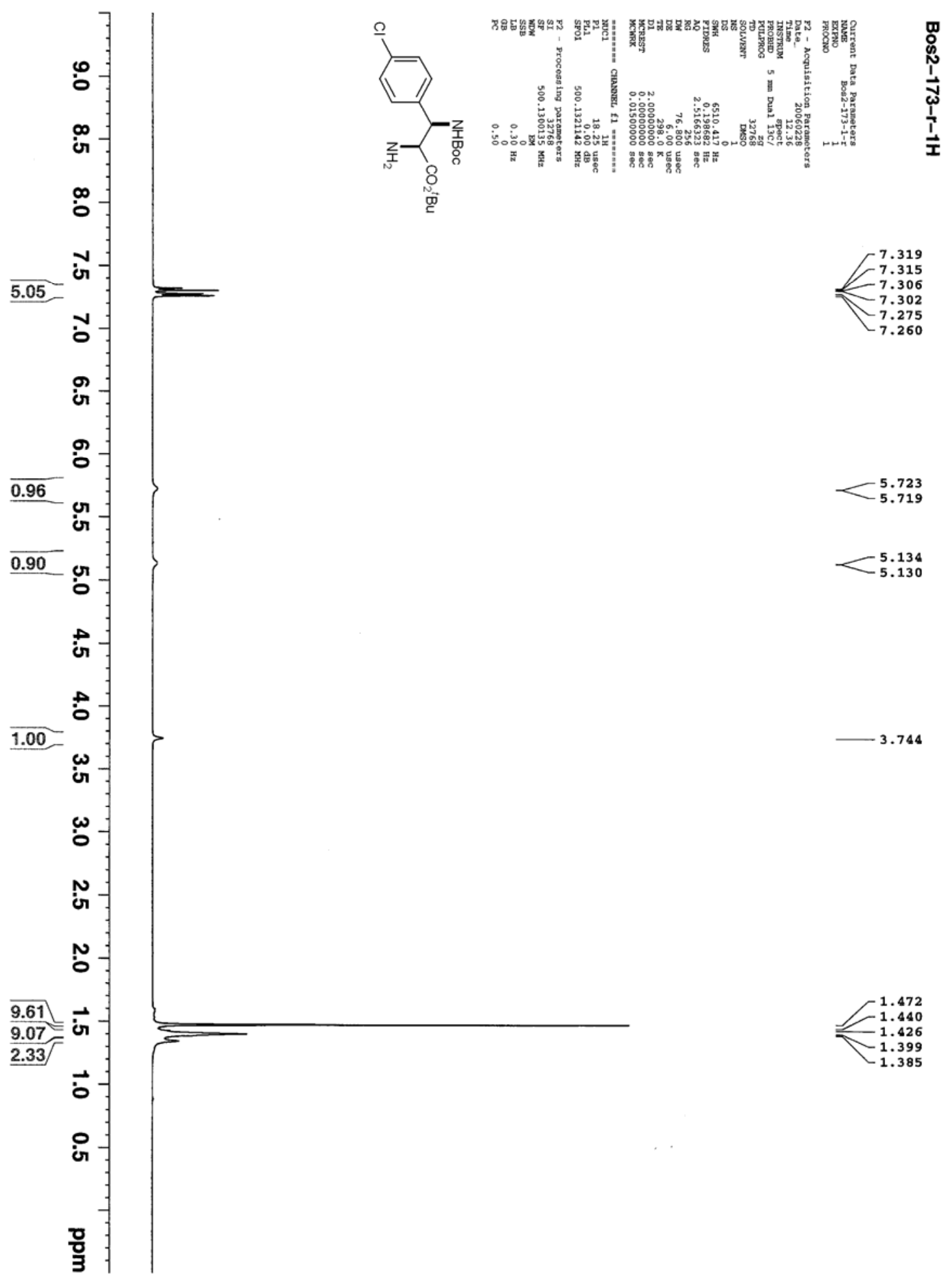




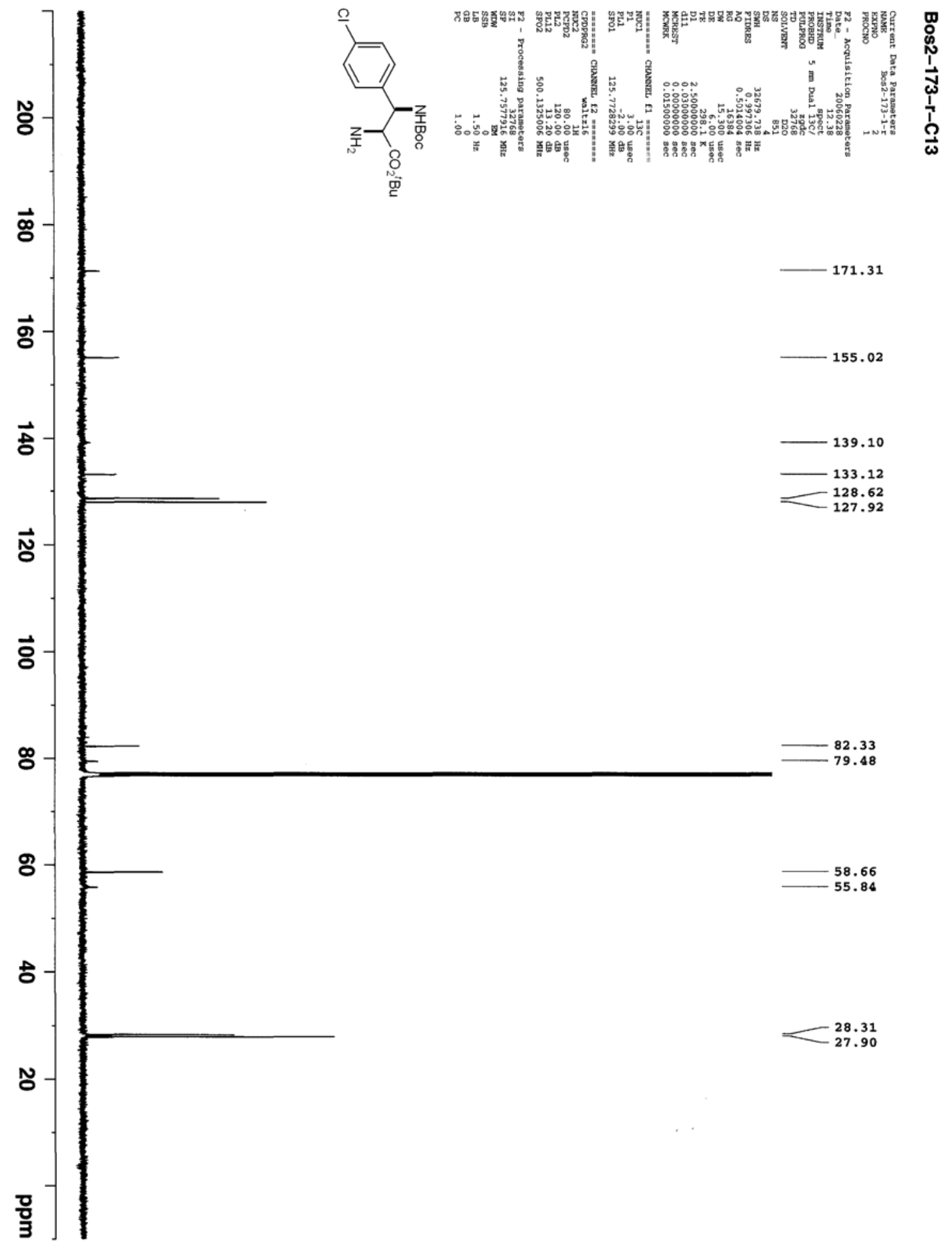




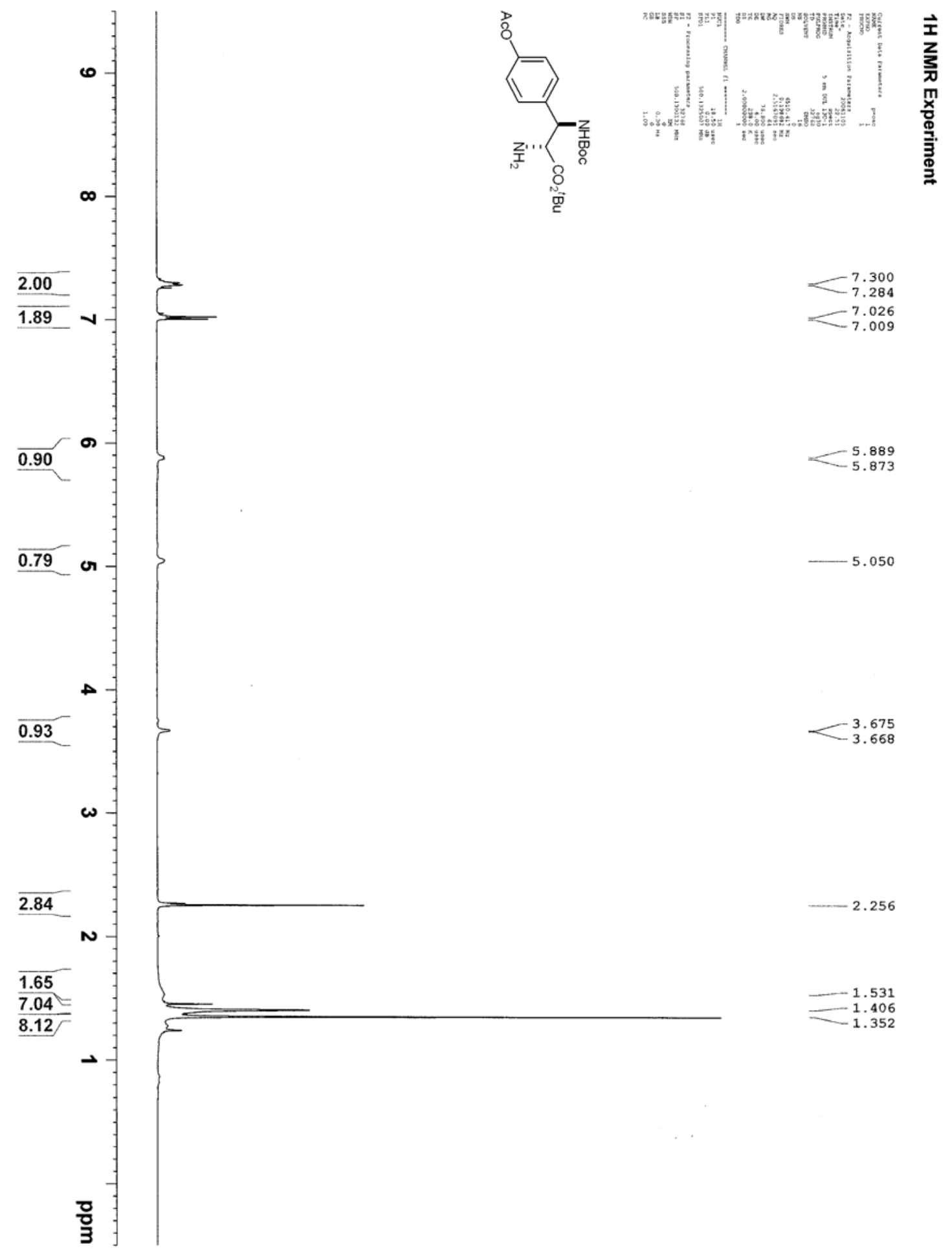




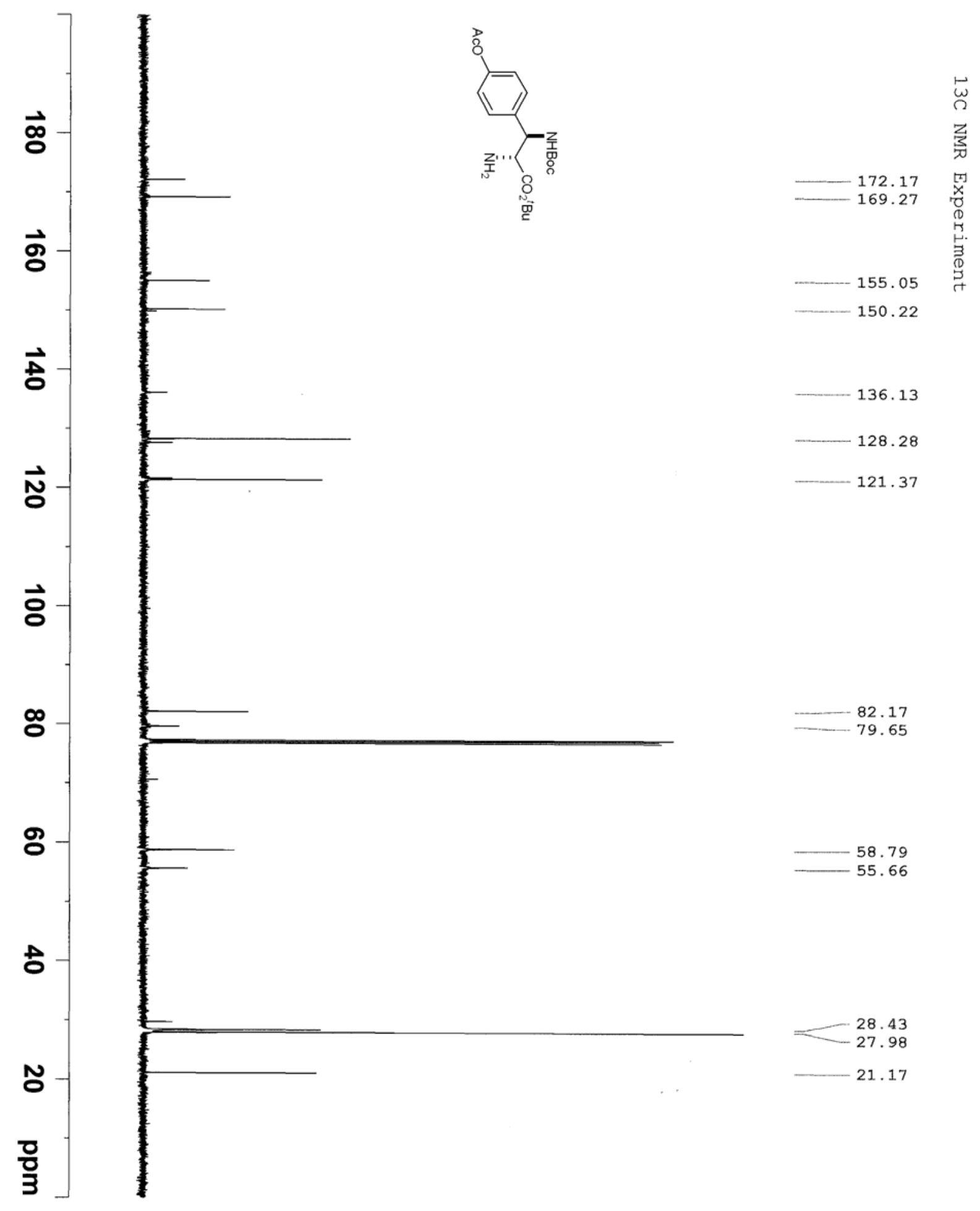




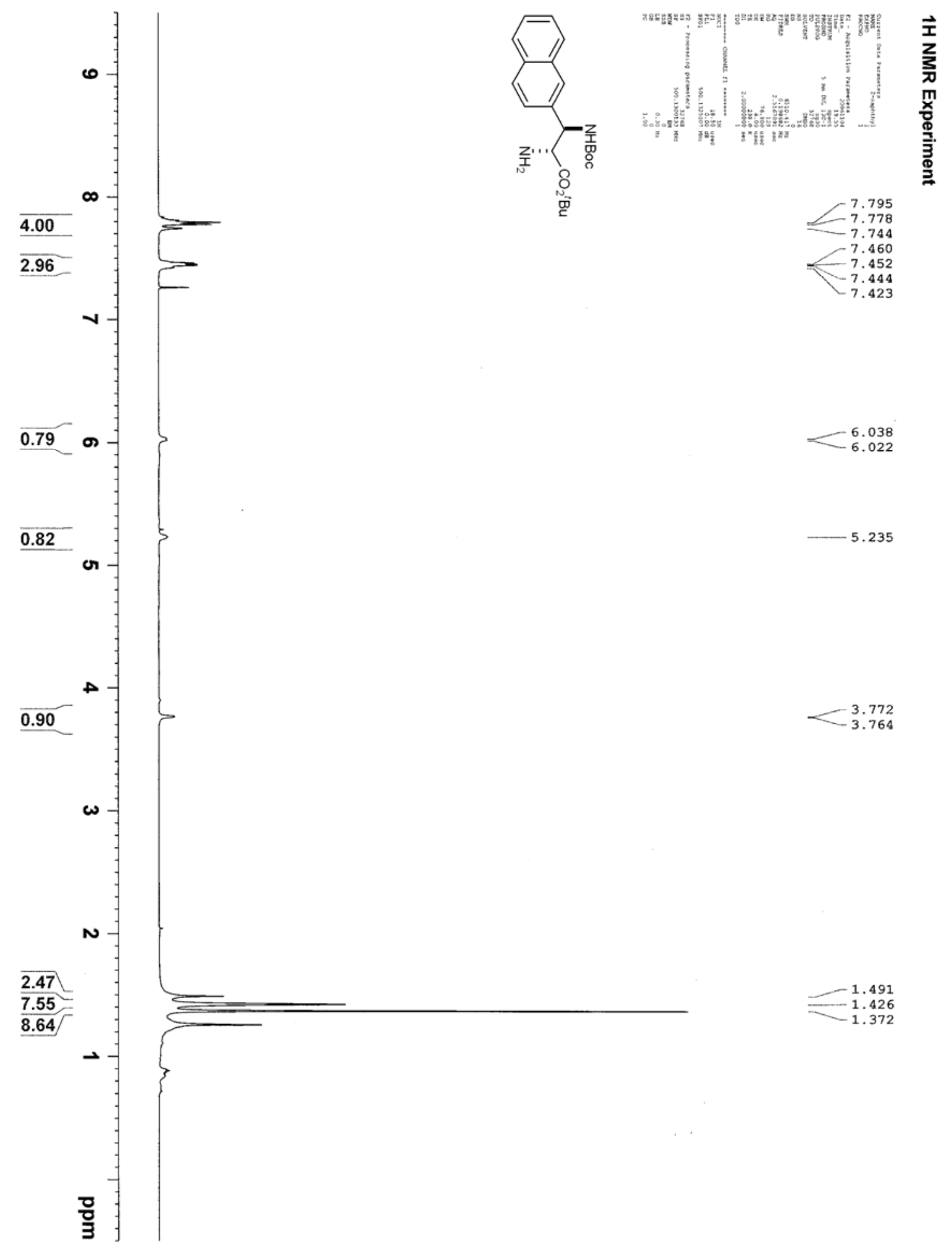




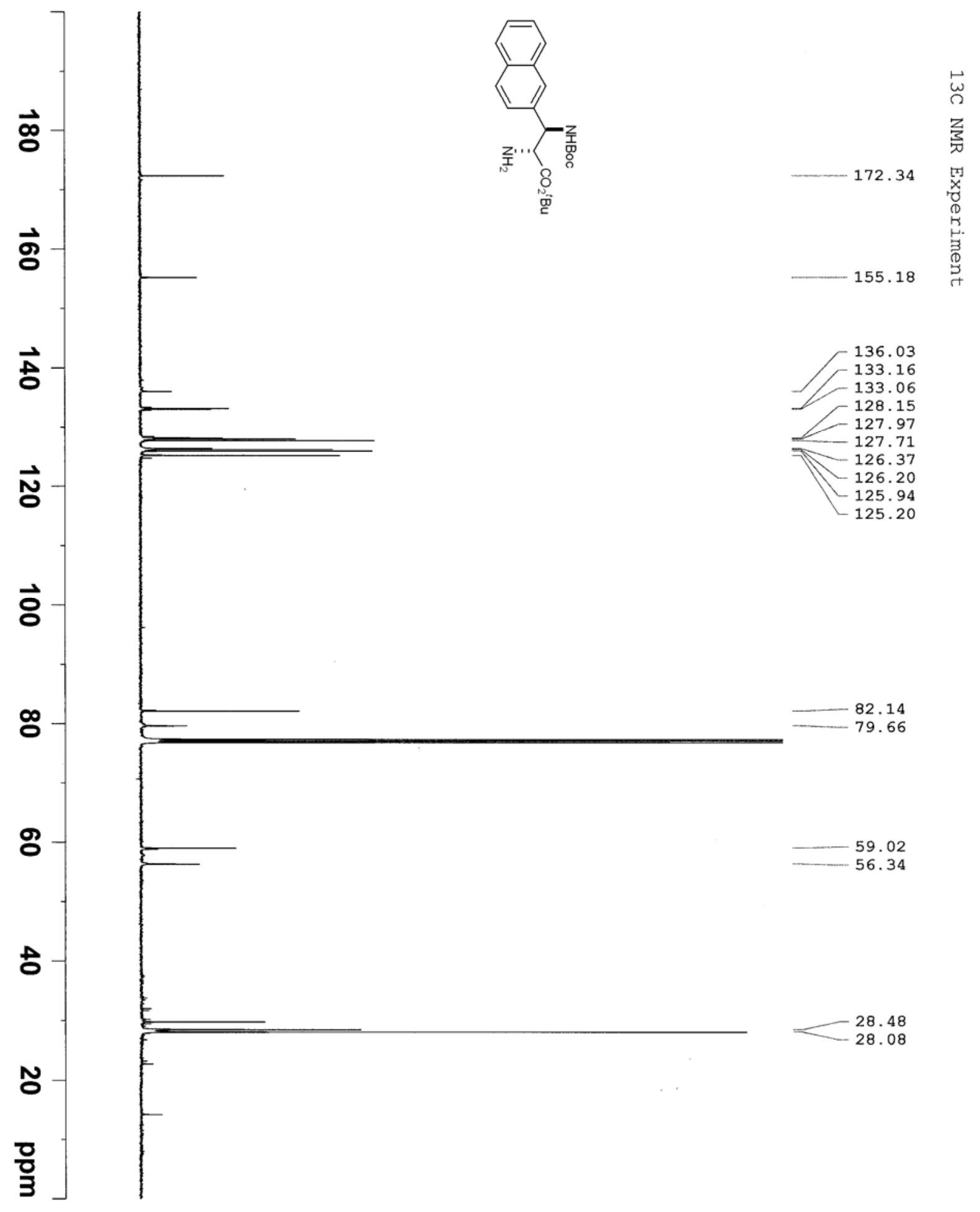




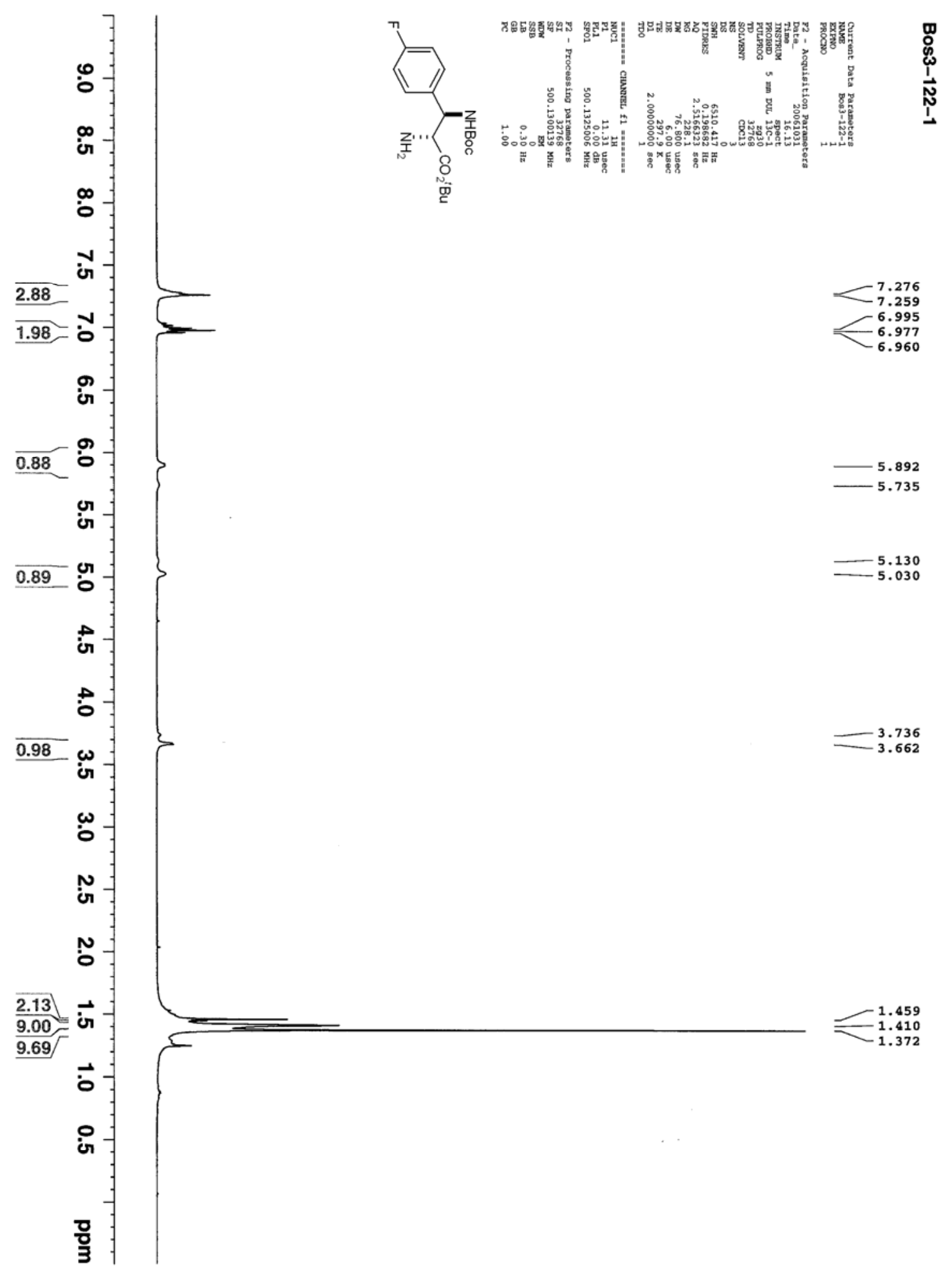




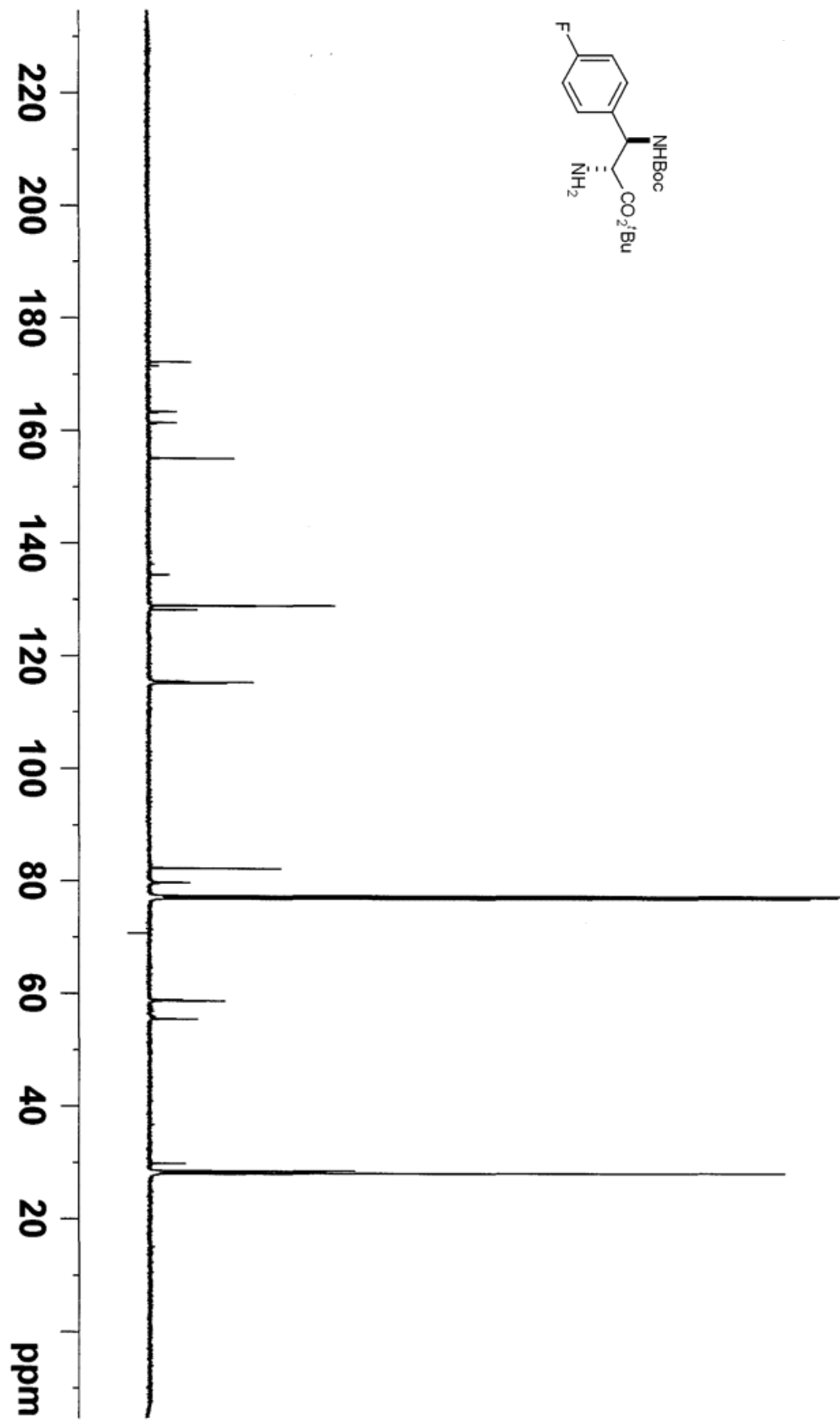

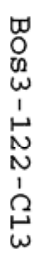

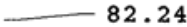

79.72

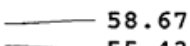

55.43

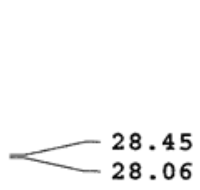




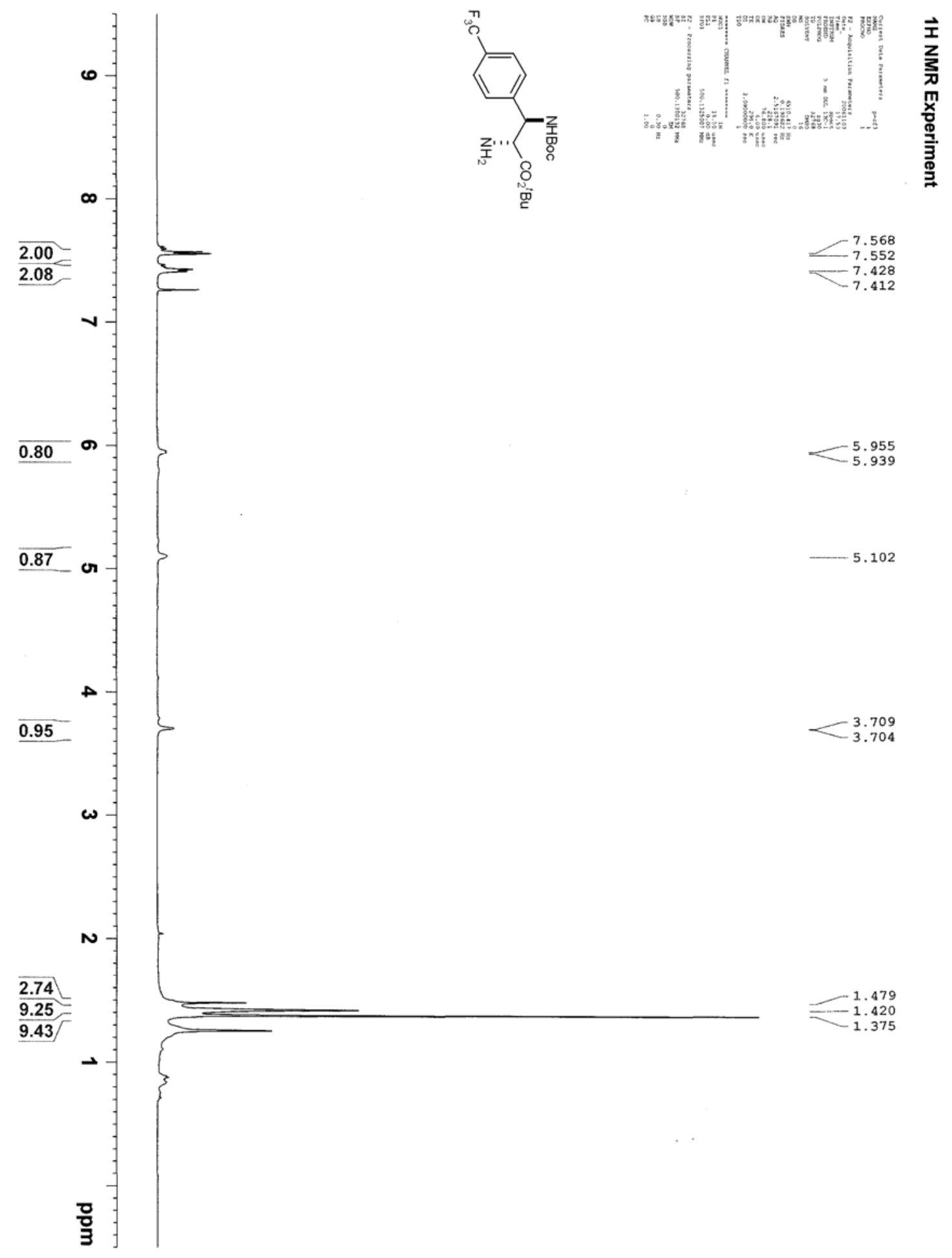




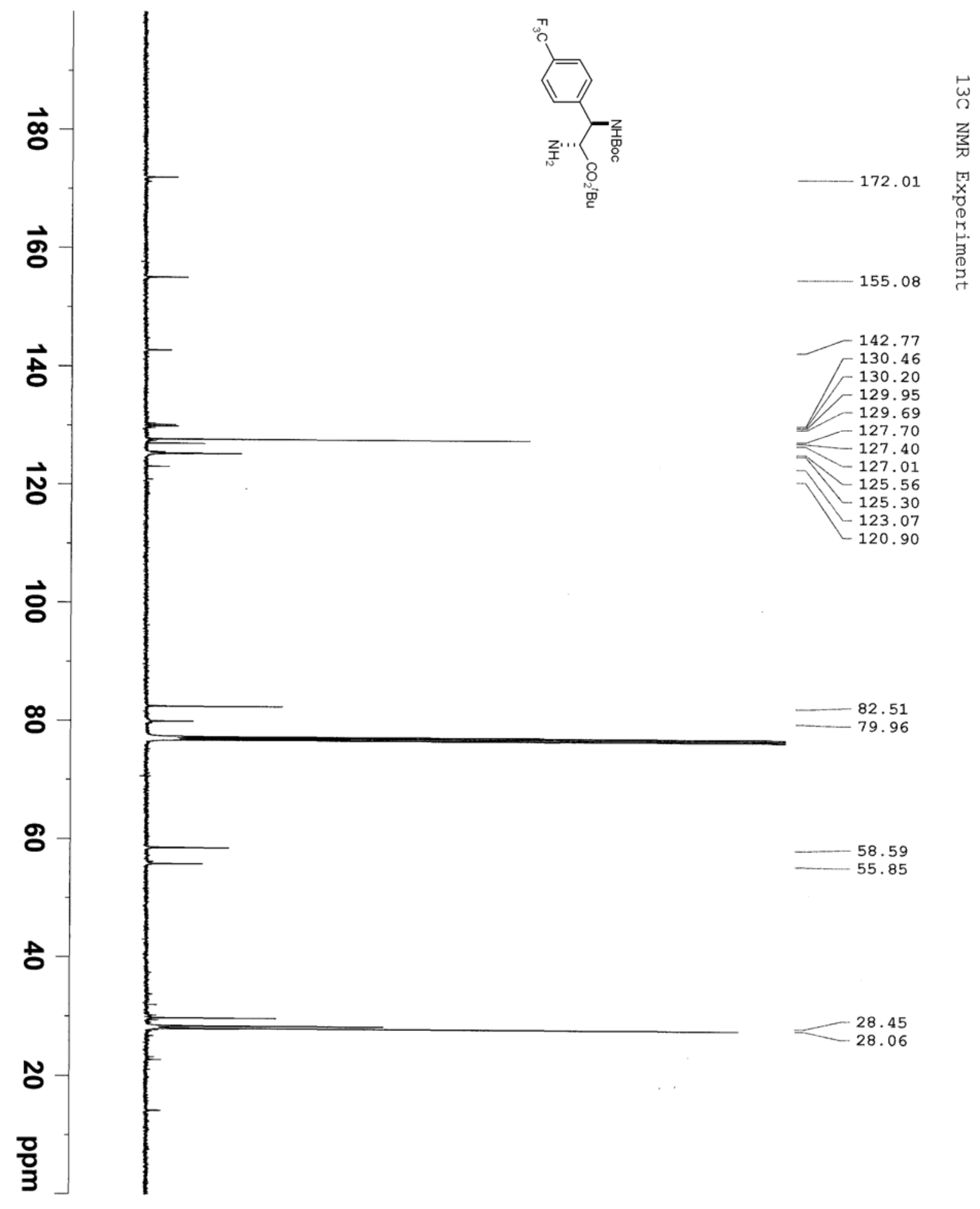




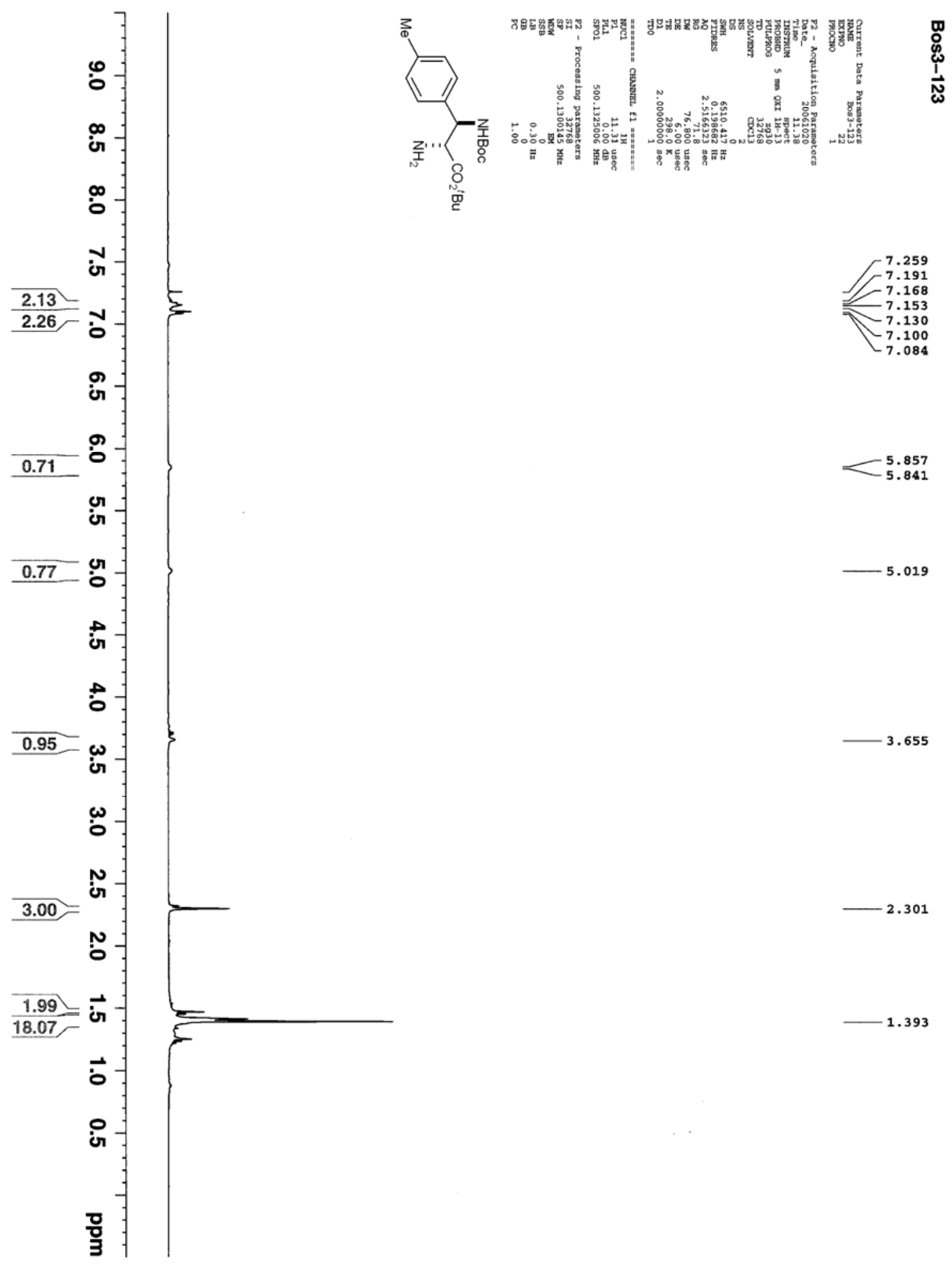




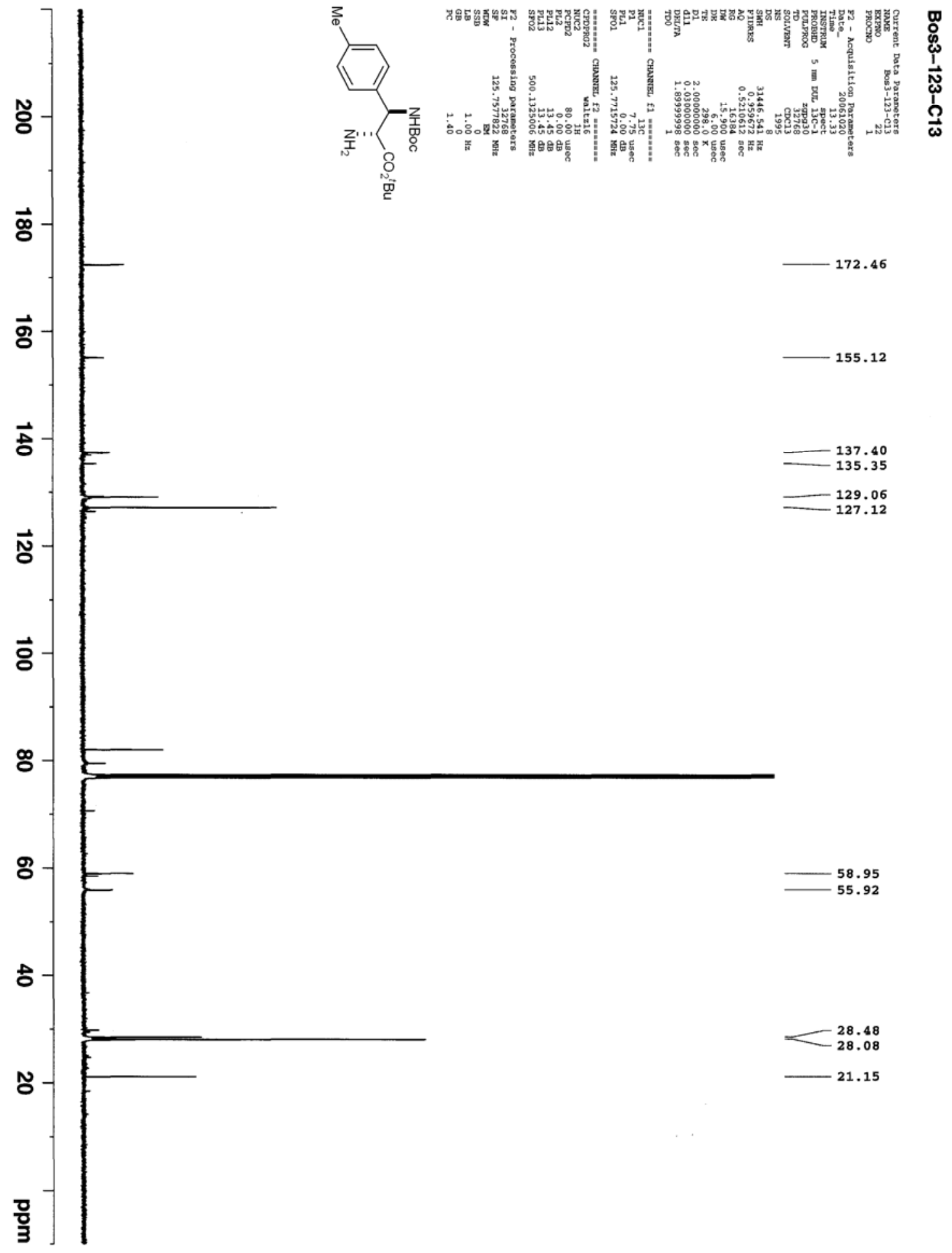




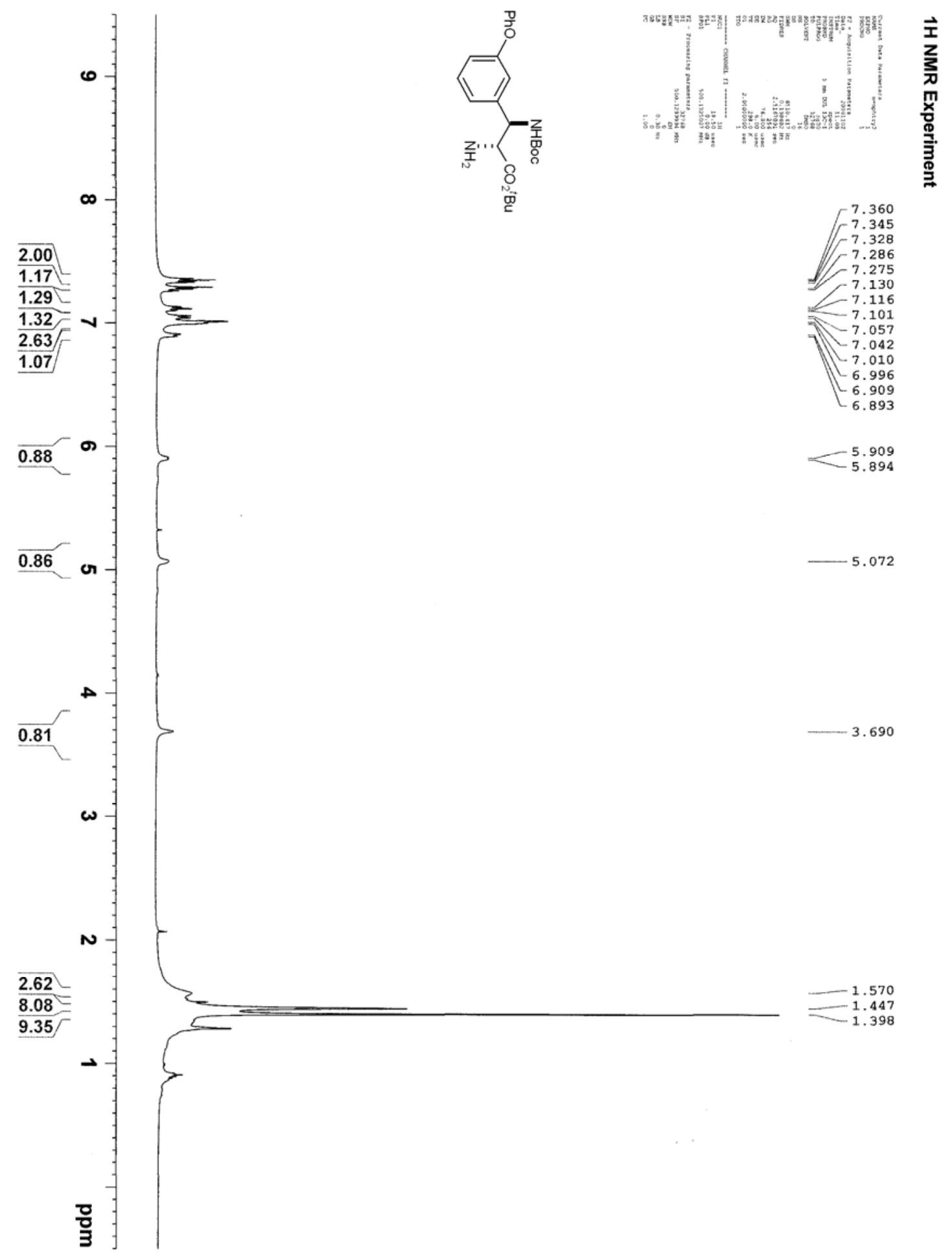




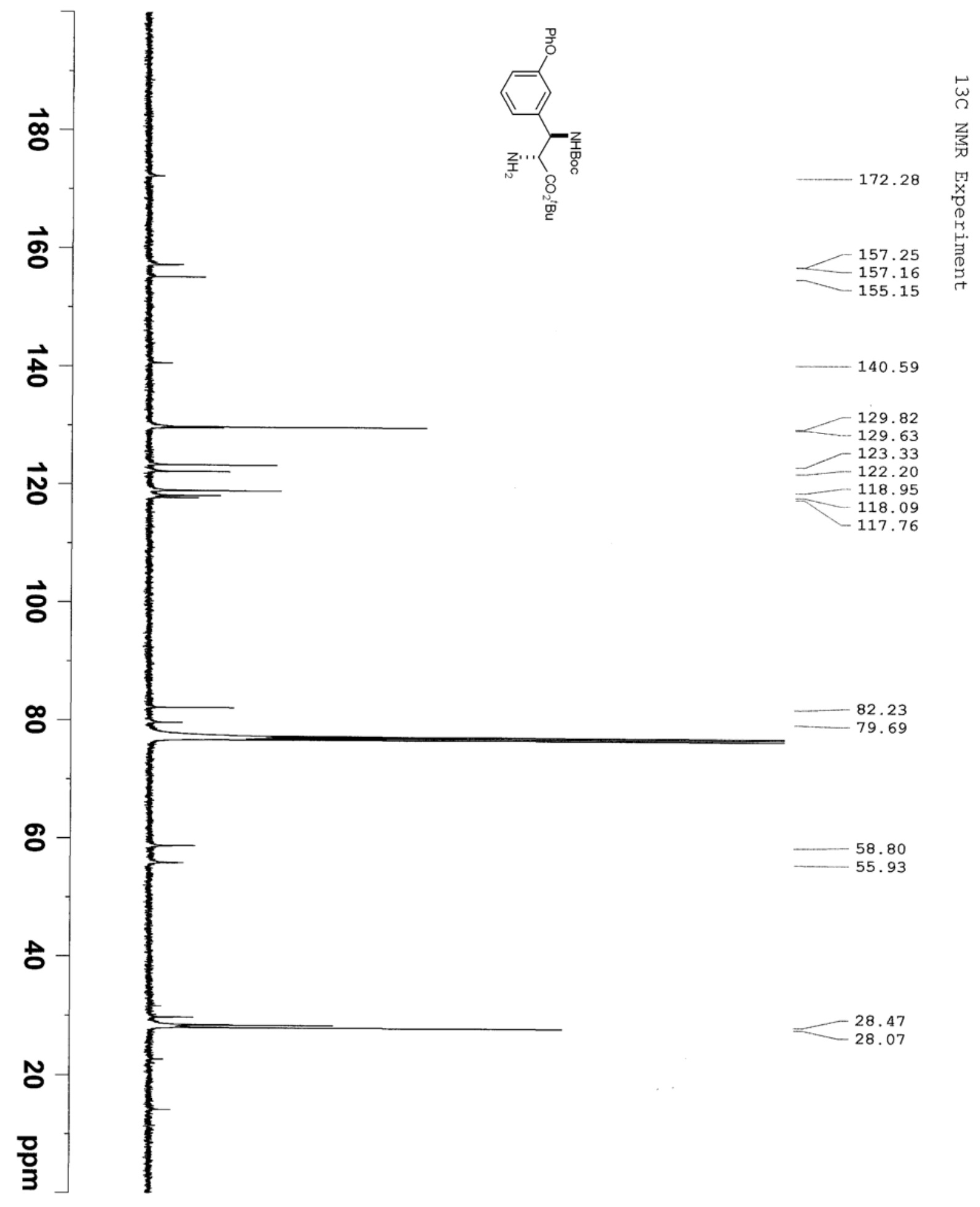




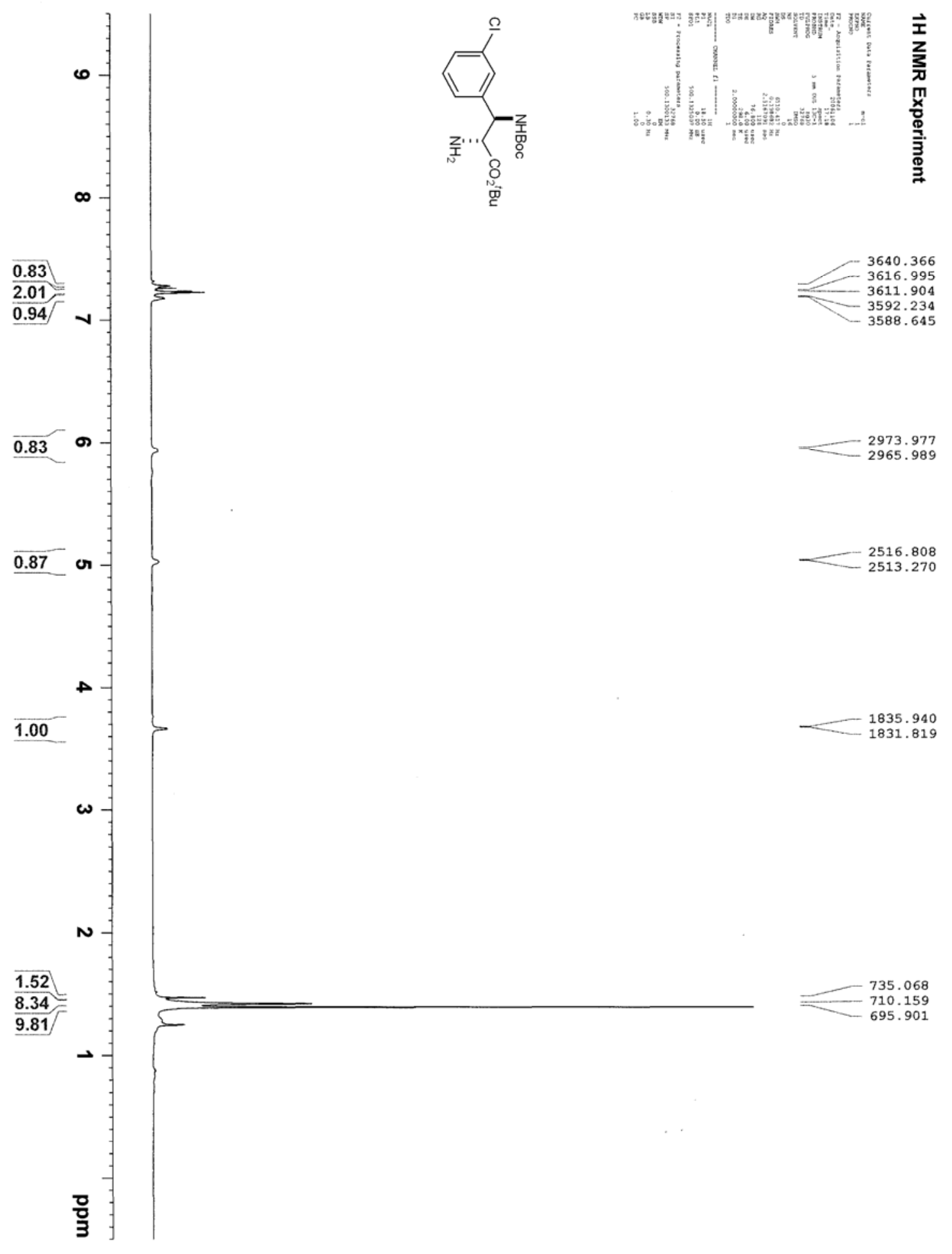




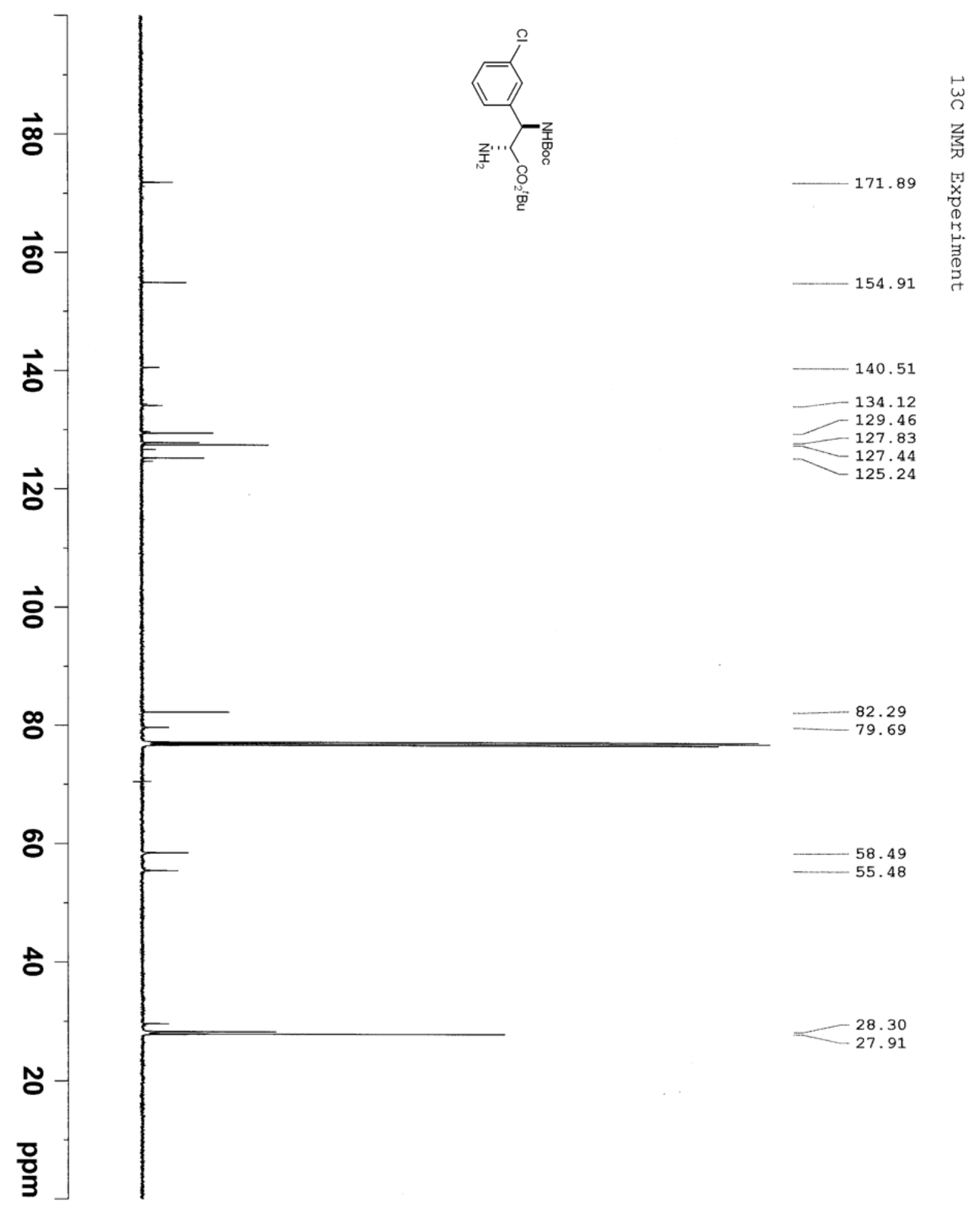




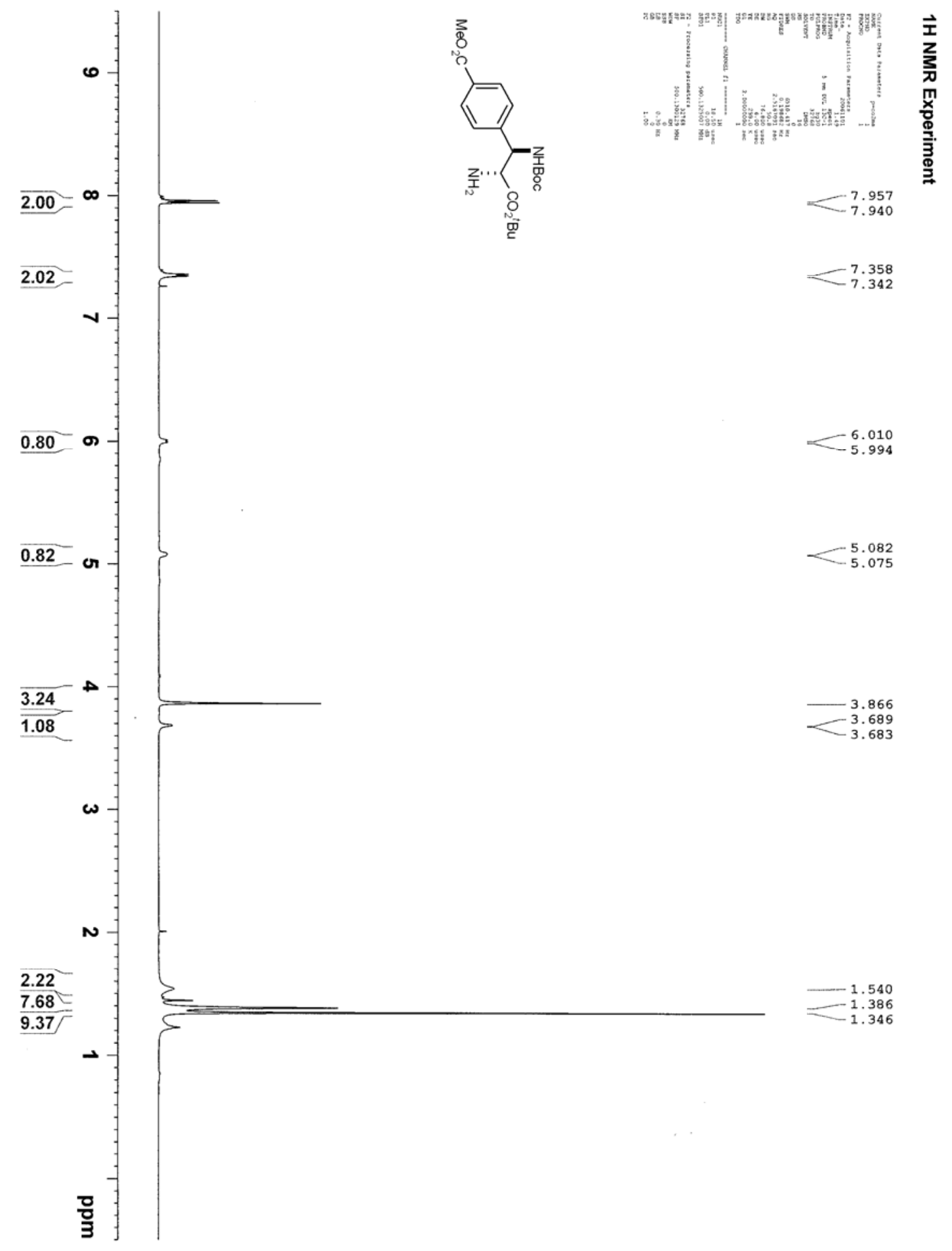




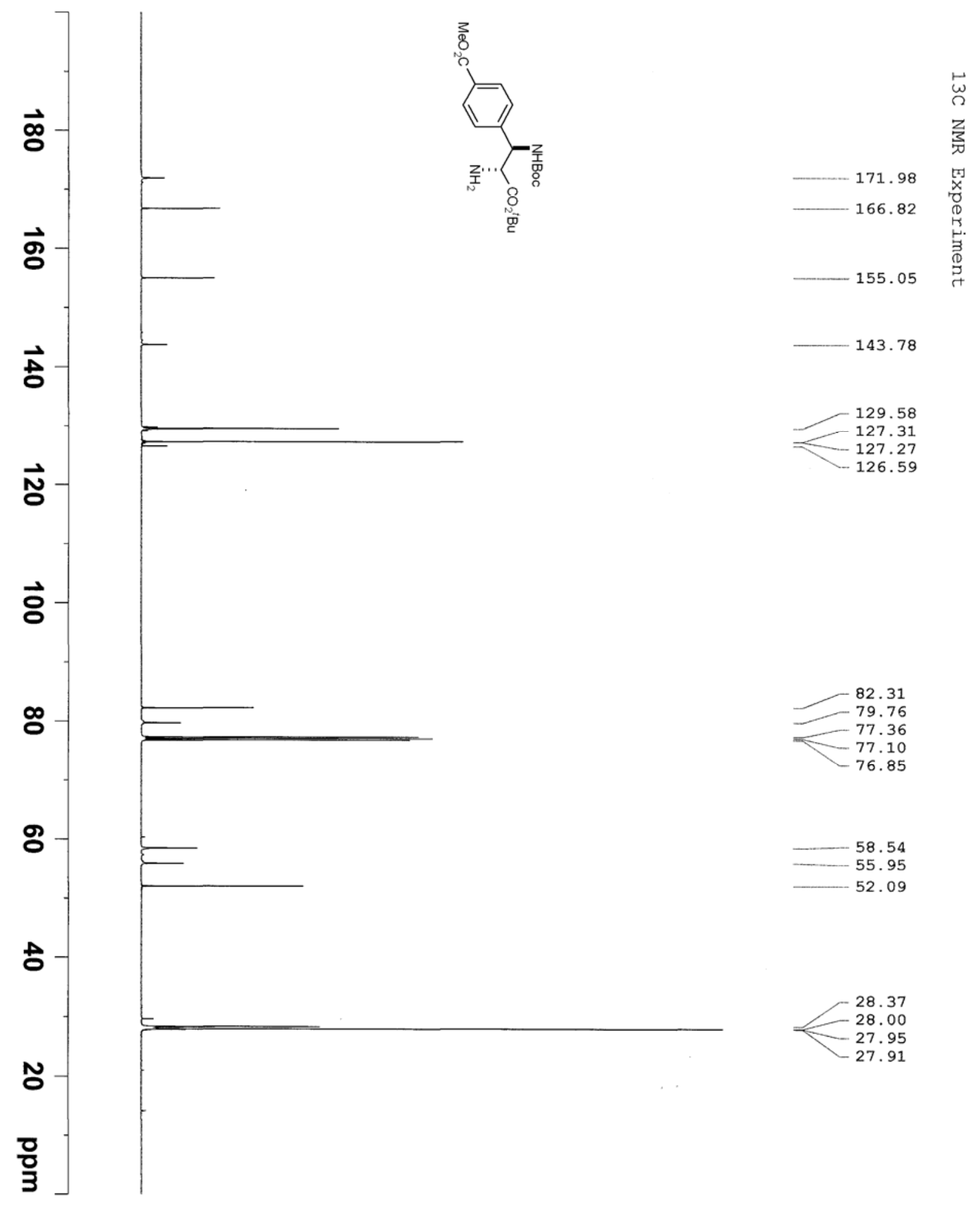



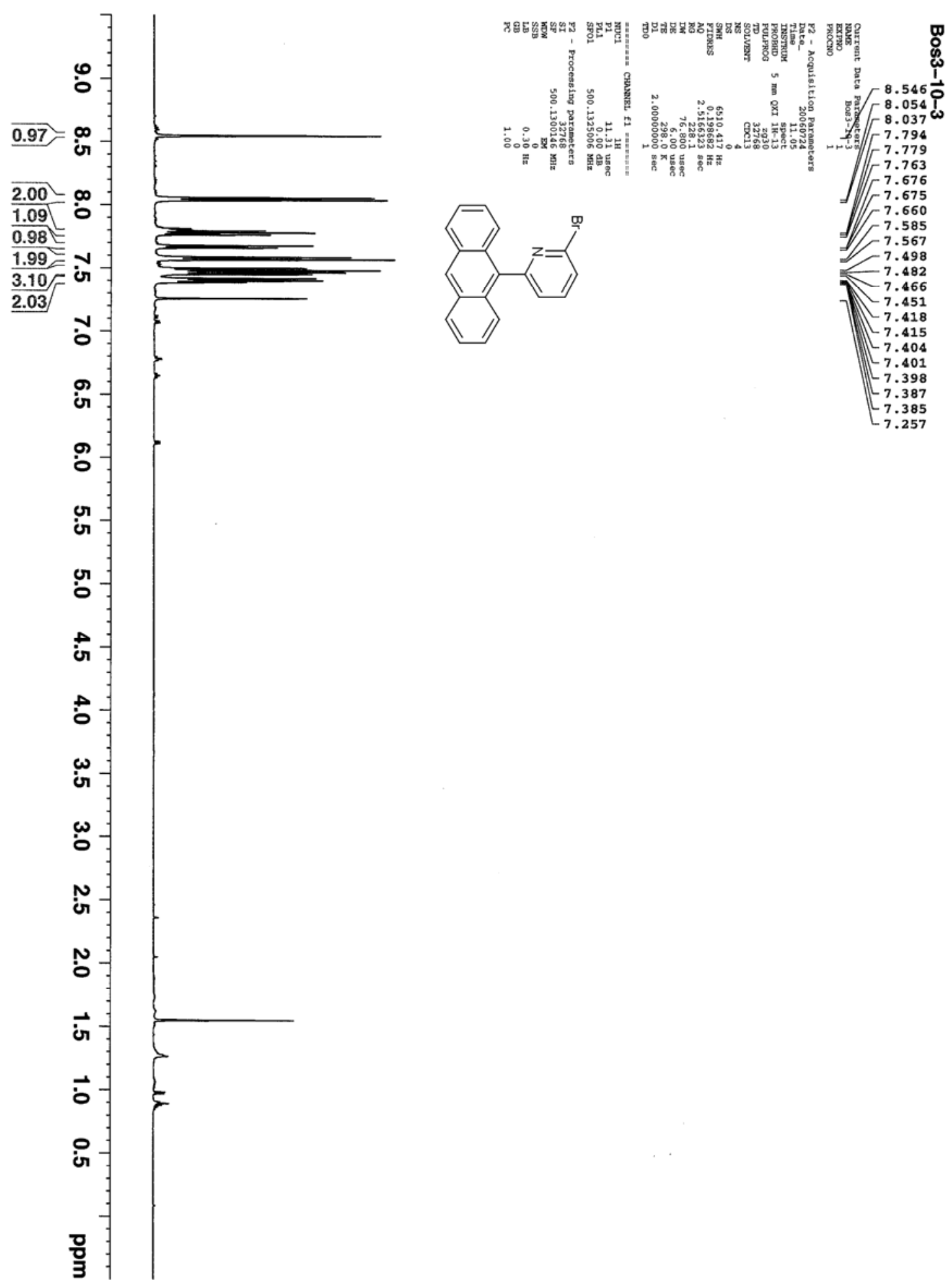


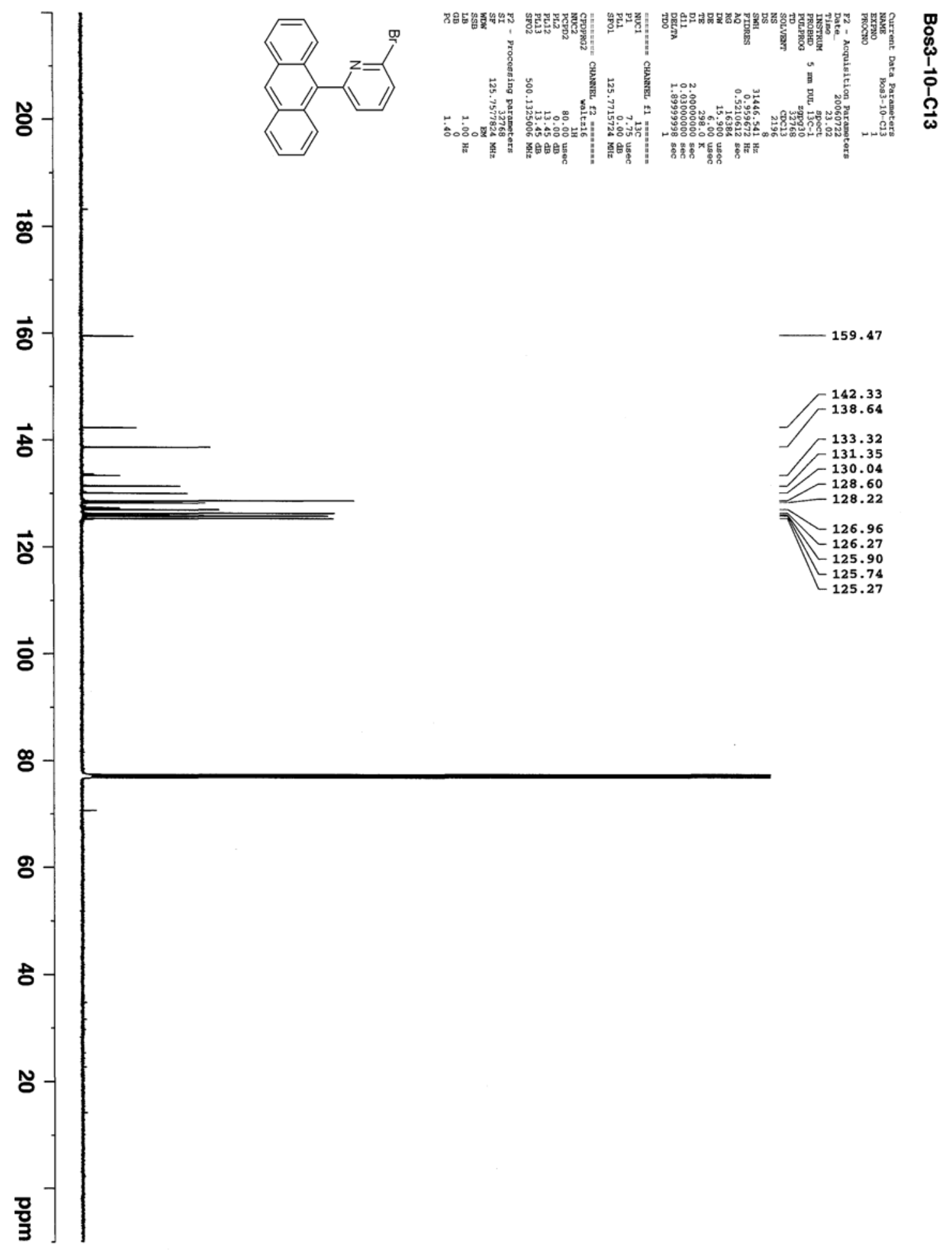




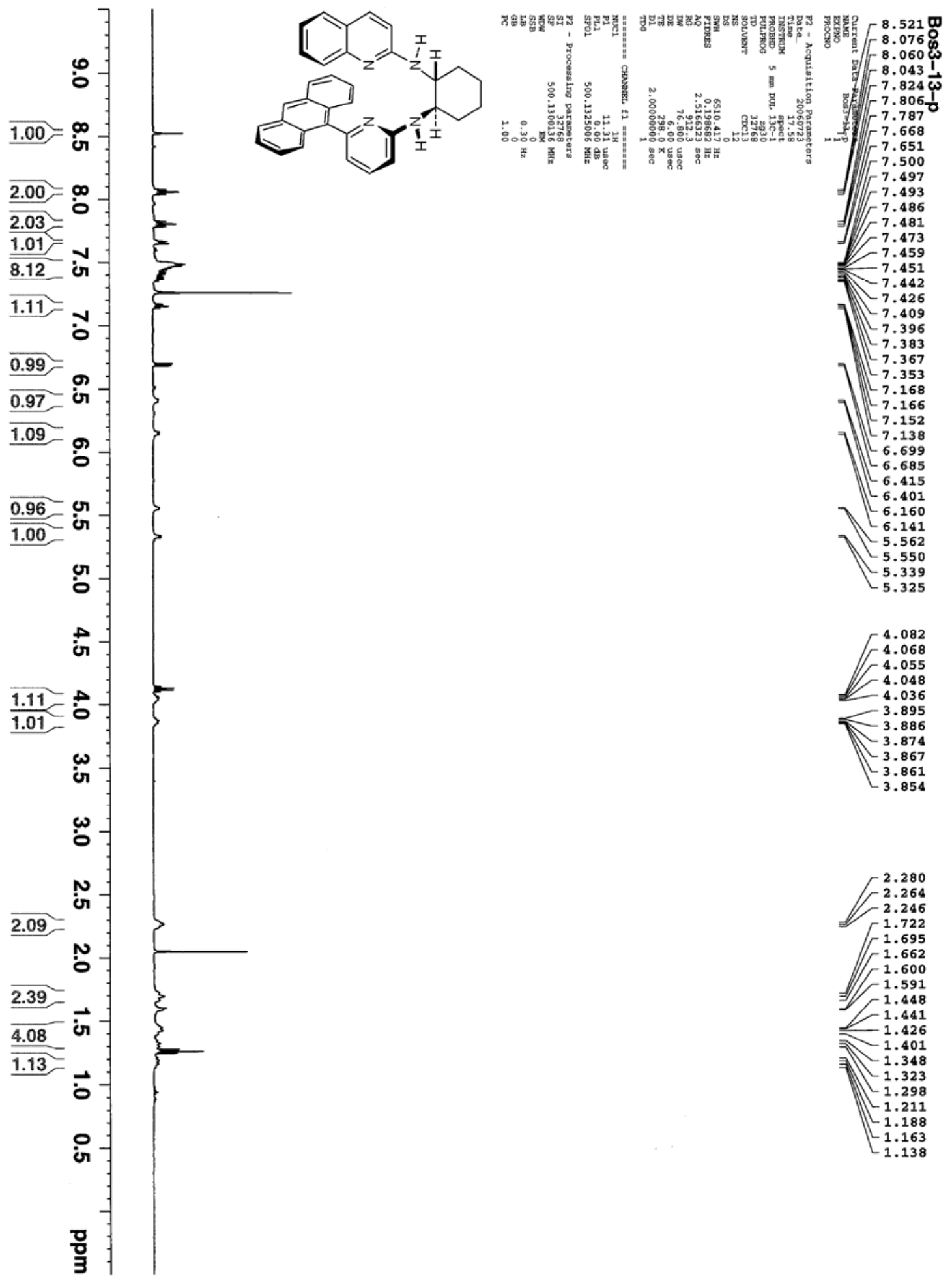




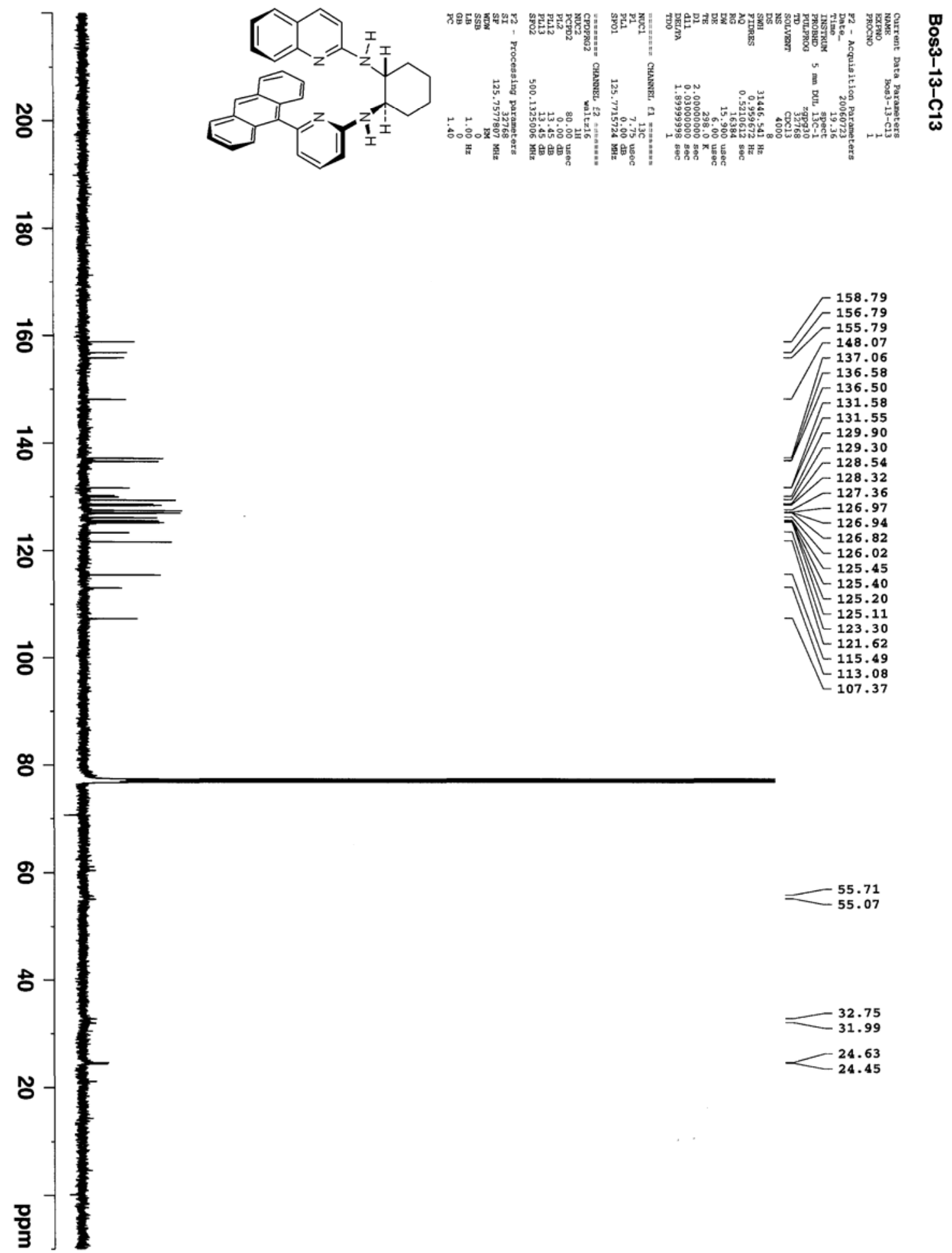

